\title{
TRPV channel OCR-2 is distributed along C. elegans chemosensory cilia by diffusion in a local interplay with intraflagellar transport
}

Jaap van Krugten, Noémie Danné, and Erwin J.G. Peterman*

LaserLaB and Department of Physics and Astronomy, Vrije Universiteit Amsterdam, De Boelelaan 1081, 1081 HV, Amsterdam, The Netherlands

*Corresponding author and Lead Contact: e.j.g.peterman@vu.nl 


\section{Abstract}

2 Sensing and reacting to the environment is essential for survival and procreation of most organisms.

3 Caenorhabditis elegans senses soluble chemicals with transmembrane proteins (TPs) in the cilia of its

4 chemosensory neurons. Development, maintenance and function of these cilia relies on intraflagellar

5 transport (IFT), in which motor proteins transport cargo, including sensory TPs, back and forth along the

6 ciliary axoneme. Here we use live fluorescence imaging to show that IFT machinery and the sensory TP

7 OCR-2 reversibly redistribute along the cilium after exposure to repellant chemicals. To elucidate the

8 underlying mechanisms, we performed single-molecule tracking experiments and found that OCR-2

9 distribution depends on an intricate interplay between IFT-driven transport, normal diffusion and

10 subdiffusion that depends on the specific location in the cilium. These insights in the role of IFT on the

11 dynamics of cellular signal transduction contribute to a deeper understanding of the regulation of sensory

TPs and chemosensing. 


\section{Introduction}

Across the tree of life, organisms depend on avoidance behavior to translocate themselves to a more favorable environment (Behringer et al., 2018; Benov and Fridovich, 1996; Gruntman et al., 2017; Hendry et al., 2018; Moore et al., 2019). In most cases, the ability to sense the outside environment is dependent on transmembrane proteins (TPs) triggering and amplifying downstream signaling pathways, including neuronal activity, that evoke a behavioral response. Primary cilia are slender protrusions of eukaryotic cells that function as signaling hubs, harboring a myriad of TPs involved in signal transduction (Brear et al., 2014; Dwyer et al., 1998; Edwards et al., 2000; Goetz and Anderson, 2010). In general, external stimuli are sensed by G-protein coupled receptors (GPCRs), which relay signals via a series of second messenger molecules like cAMP and calcium to elicit a behavioral or genomic response (Nachury and Mick, 2019; Schou et al., 2015). The ciliary distribution of these TPs is essential for their function, and their location is regulated by the intraflagellar transport (IFT) machinery (Blacque et al., 2004; Mukhopadhyay et al., 2010). Previous work on the dynamics of GPCR's in mammalian cell lines has shown that they mostly move by passive diffusion, although active transport is observed, most notably during signal-dependent retrieval of GPCR's out of the cilia (Ye et al., 2018).

The nematode $C$. elegans has proven to be an invaluable model organism to study proteins involved in signal transduction and has been widely used as a model for avoidance behavior and the dynamics of ciliary TPs (Perkins et al. 1986; Culotti and Russel, 1978; Jansen et al. 1999; Hilliard et al. 2004). Particular emphasis has been on $C$. elegans sensory cilia, which are located at the end of dendrites of chemosensory neurons in the amphid and phasmid channels, situated in the head and tail regions of the worm, respectively. Mutagenesis studies, combined with experiments where worms are exposed to a droplet of a chemical compound, have demonstrated that $C$. elegans shows chemotaxis and avoidance behavior and have allowed identification of the neurons involved (Culotti and Russell, 1978; Tran et al., 2017; Ward, 1973). Attractants are sensed by the amphid neurons, while the response to repellents appears to be modulated by a combination of the phasmid neurons and amphid neurons (Hilliard et al., 2002). Together, the sensory neurons and cilia of $C$. elegans allow the nematode to react to environmental stimuli within seconds (Ward, 1973).

Development, maintenance and function of cilia critically depend upon a specific transport mechanism, IFT. IFT consists of transport units called IFT trains, that travel from ciliary base to tip and back again, facilitating transport of ciliary building blocks and sensory proteins (Scholey, 2003). In C. elegans cilia, anterograde transport (from base to tip) is driven by heterotrimeric kinesin-II in the first few micrometers of the cilium, after which it gradually hands over the IFT trains to homodimeric kinesin-2 OSM-3 (Prevo et al., 2015; Signor et al., 1999). At the tip of the cilium, IFT trains disassemble and remodel (Mijalkovic et al., 2018). Retrograde transport, back to the base is driven solely by IFT dynein (cytoplasmic dynein-2) (Engel et al., 2012; Mijalkovic et al., 2017; Pazour et al., 1999).

The backbone of these trains is formed by the IFT-A, IFT-B and BBSome particle complexes. The link between IFT trains and functional cargo like GPCRs is most likely formed by Tubby proteins and the BBSome. Tubby-like protein 3 (TULP3), together with IFT-A, was found to be essential for the ciliary import of some GPCRs (Badgandi et al., 2017; Mukhopadhyay et al., 2010). Mutations in the BBSome and the C. elegans TULP3 homolog, tub-1, show chemotaxis defective behavior, demonstrating the 
importance of the localization and active transport of sensory TPs (Blacque et al. 2004, Mukhopadhyay et al., 2005).

Electron microscopy and experiments with fluorescent beads tethered to membrane glycoproteins in Chlamydomonas have shown directly that IFT trains are physically linked to the membrane (Pigino et al., 2009; Shih et al., 2013). Studies in mouse, Chlamydomonas and C. elegans have demonstrated that, among other cargoes, several types of TPs are transported by IFT trains (Lechtreck, 2015). Ensemble fluorescence microscopy studies have shown movement by IFT of the ion-channels PKD2 and OCR-2, in Chlamydomonas and C. elegans respectively (Huang et al., 2007; Qin et al., 2005). Elegant single-molecule studies in cultured mammalian cells demonstrate ciliary TPs exhibiting complex dynamics of both passive diffusion and active transport by IFT (Milenkovic et al., 2015; Weiss et al., 2019; Ye et al., 2013). While occasionally undergoing active transport, ciliary receptors like Smoothened were found to be frequently confined at the ciliary base, and to mostly exhibit passive diffusion in the ciliary membrane.

Despite this progress on the understanding of the transport mechanisms that transport TPs in cilia many questions remain. It is, for example, unclear what the interplay is between active, IFT-driven, and passive, diffusive transport of TPs, how this is regulated and whether it depends on stimulation of the cilia. To shed light on this, we investigate here the dynamics underlying the redistribution of ciliary components during avoidance behavior in the chemosensory cilia of live $C$. elegans using sensitive fluorescence microscopy. With the goal to connect the whole-organism avoidance behavior to the single-molecule dynamics of the proteins at the basis of sensory signal transduction. We find that repellent addition results in a reversible redistribution of the IFT machinery, the ciliary axoneme, and the TRPV cation channel OCR-2 out of the ciliary distal segment (DS). To elucidate the dynamics underlying this redistribution, we performed single-molecule fluorescence microscopy of OCR-2 in the phasmid cilia of live C. elegans. Analysis of the trajectories reveals that active, IFT-driven transport is the predominant mode of transport in the dendrite and transition zone. Along the cilia, the proper distribution of OCR-2 depends on an intricate location-specific interplay between active transport, normal diffusion and subdiffusion, with the latter playing an important role in the confinement of TPs at the ciliary tip. These insights in the dynamics of cellular signal transduction during external stimulation contribute to a wider understanding of IFT dynamics and to cilia as chemosensory organelles triggering behavioral responses. 

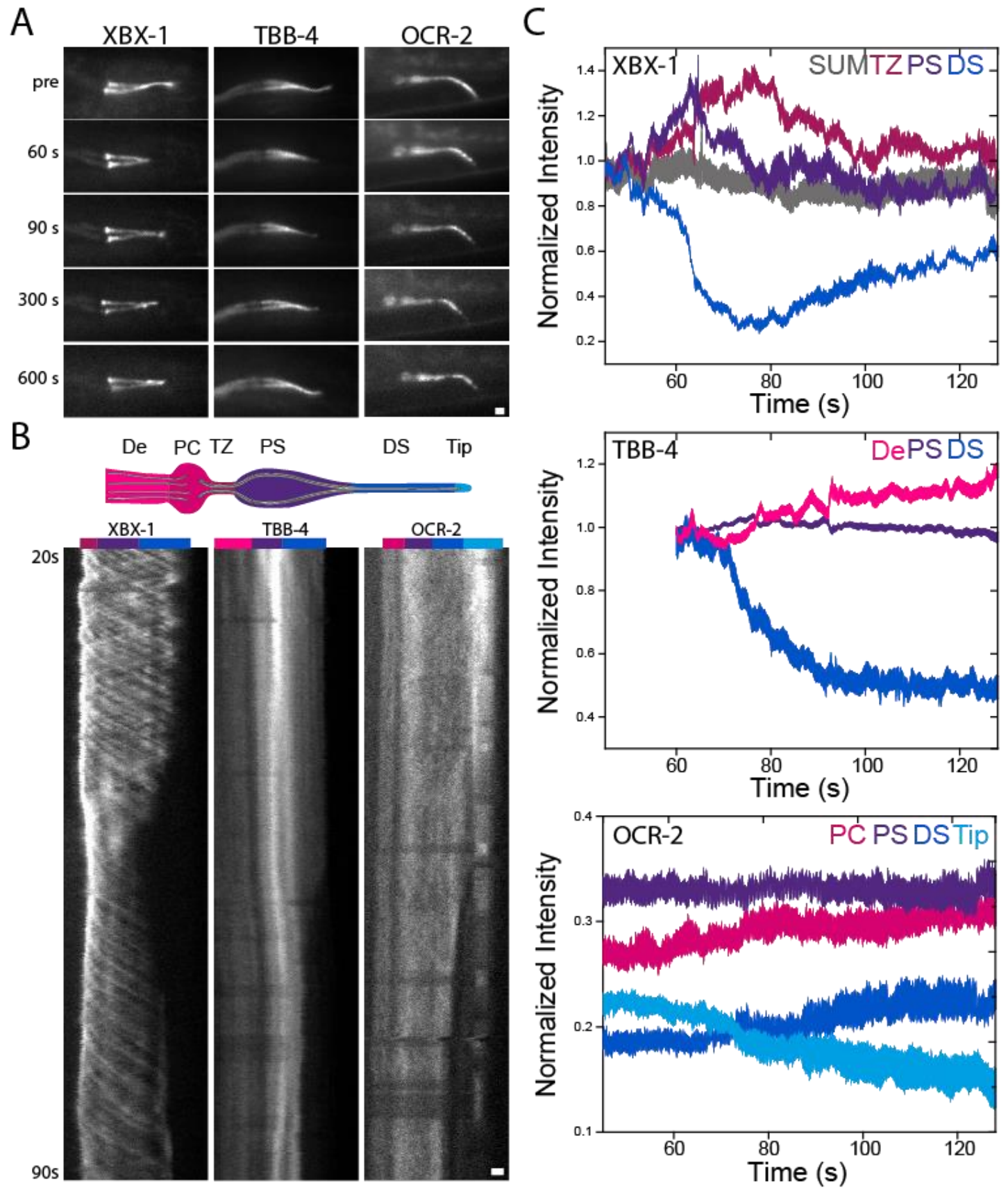

Figure 1: Ciliary components redistribute reversibly upon SDS exposure. (A) Time-averaged (over 3 s) fluorescence images of TBB-4::EGFP, XBX-1::EGFP and OCR-2::EGFP before and after SDS exposure. Scale bar: $1 \mu \mathrm{m}$. (B) Cartoon illustrating the ciliary segments (PCMC: periciliary membrane compartment; TZ: transition zone; PS: proximal segment; DS: distal segment) and representative kymographs of TBB-4::EGFP, XBX-1::EGFP and OCR-2::EGFP starting $20 \mathrm{~s}$ after SDS exposure. Scale bar: $1 \mu \mathrm{m}$. (C) Relative fluorescence intensities of TBB-4::EGFP, XBX-1::EGFP and OCR-2::EGFP in different ciliary segments. For TBB-4::EGFP and XBX-1::EGFP, normalized by the average fluorescence intensities of the first 10 frames. For OCR-2::EGFP, the total fluorescence intensity along the whole cilium was normalized, immediately after SDS exposure. 


\section{Results and discussion}

\section{Exposure to repellents leads to reversible redistribution of IFT components}

Exposing C. elegans to certain chemicals, including SDS, leads to a drastic change in behavior: the worm abruptly ceases its elegant, sinusoidal motion and changes direction. This behavior is called the avoidance response and is triggered by its sensory cilia (Perkins et al., 1986). The nervous system of C. elegans consists of 302 neurons, of which 32 are ciliated. Most of these cilia are situated in the two amphid openings, in the head region of the nematode. Because of their number, and the high background fluorescence around the amphid cilia, we study the two pairs of phasmid cilia (PHAL, PHAR, PHBL and PHBR), located in the tail of the animal. To obtain insight in the molecular response of the phasmid chemosensory cilia to aversive compounds, we added water-soluble repellant chemicals while imaging the cilia using epi-illuminated widefield fluorescence microscopy. Strains endogenously expressing fluorescently labeled TBB-4 (TBB-4::EGFP) as marker for axonemal microtubules and XBX-1 (XBX1::EGFP) (Mijalkovic et al., 2017), an IFT-Dynein marker that allows probing IFT dynamics, were exposed to $0.1 \%$ (W/V) sodium dodecyl sulfate (SDS), by pipetting a droplet on them. SDS is thought to be a chemical mimic of dodecanoic acid, a compound secreted by nematicide-secreting Streptomyces bacteria (Tran et al., 2017). C. elegans has been demonstrated to avoid SDS, even at a concentration as low as $0.1 \%$, which is below its critical micelle concentration (Rahman and Brown, 1983). To test the spreading of SDS, the fluorescent compound fluorescein was added in an identical way. This experiment indicates that nematodes, when exposed to a droplet of dissolved chemical compound, experience an immediate increase of compound concentration, which settles to a maximum concentration after two minutes (Figure S1).

Even though worms had been anesthetized with $5 \mathrm{mM}$ Levamisole, they still exhibited an avoidance response within seconds after SDS addition, as evident from a sudden movement of the organisms (Movie S1). We set this particular moment in time to zero. This movement, together with the adverse effect of droplet addition on imaging, made constant refocusing necessary, rendering the frames of the first tens of seconds after addition impossible to interpret in most cases. To visualize the distribution of the fluorescent ciliary components, we time-averaged (over $3 \mathrm{~s}$ ) subsequent fluorescence images such as the one shown in (Figure 1A, Movie S1). First, we focus on IFT Dynein. The images show that, before SDS addition, IFT dynein occupies the entire length of the phasmid cilia, as observed before (Mijalkovic et al., 2017). After about a minute, the motors have drastically redistributed, extending only from the base of the cilium up to the end of the middle segment. At longer time scales the distribution appears to have recovered completely to the original situation, before applying the stimulus.

To get more insight in the redistribution dynamics, we generated kymographs from the original fluorescence image sequences (Mangeol et al., 2016) (Figure 1B). The colored bars at the top of the kymographs indicate the dendrite, PCMC and ciliary segments, as schematically represented in the cartoon on top. The kymograph shows that, up to about a minute after SDS exposure, processive IFTdynein trains can be observed moving from ciliary base to tip. After a minute, however, IFT dynein suddenly redistributes out of the distal segment (DS), no longer reaching the ciliary tip, but recovers its pre-exposure distribution quickly after. 
To quantitatively compare the amounts of proteins in the different ciliary segments in several worms, the average fluorescence intensity per segment is shown as a function of time (Figure 1C; color coding of the ciliary segments as in cartoon). These time traces show that the amount of IFT dynein in the DS rapidly (within $\sim 20$ seconds) decreases about five-fold, $\sim 1$ minute after SDS addition. The traces indicate that IFT dynein moves away from the distal segment (DS), through the proximal segment (PS), towards the transition zone (TZ) and base (Figure 1B and C). The total amount of IFT dynein does not seem to change, indicating that the motors do not leave the cilium on this time scale. Whereas the avoidance reaction of the worms occurs within seconds after SDS addition, IFT dynein reacts later, after $49.7 \pm 4.7 \mathrm{~s}$ (mean \pm s.e.m.; $\mathrm{n}=8$ worms). This remarkable delay, between avoidance response and IFT-dynein redistribution, will be discussed further below. Taken together these result show that, about a minute after exposure to SDS, IFT dynein reversibly redistributes, temporarily leaving the DS towards the PS and ciliary base.

IFT is required for the maintenance of the cilium, and transports, among others, tubulin towards the tip of the ciliary axoneme (Kozminski et al., 1993; Marshall and Rosenbaum, 2001). To test whether the depletion of IFT dynein in the distal segment after SDS exposure leads to axoneme instability, we next imaged worms expressing fluorescent tubulin. The time-averaged fluorescence images and kymograph of TBB4::GFP (Figure 1A, B and C) show a similar redistribution as observed for IFT dynein, albeit slightly later, $68.6 \pm 6.7 \mathrm{~s}$ (mean \pm s.e.m.; $\mathrm{n}=7$ worms)). This disappearance of TBB-4 from the end of the cilium is most likely caused by shortening of the ciliary axoneme. Remarkably, the shortening appears to stop at the end of the PS, where the axoneme consists of microtubule doublets. In a previous study, femtosecondlaser ablation of the phasmid-neuron dendrites was shown to also cause the collapse of the DS, but not the PS, suggesting that the PS, consisting of microtubule doublets, is more stable than the DS, which consists of microtubule singlets (Mijalkovic et al., 2020). In contrast to IFT dynein, part of the tubulin appears to leave the cilium, as is evident from the increased TBB-4 fluorescence in the dendrite (Figure 1C). The -19 $s$ delay between the onset of TBB- 4 and IFT dynein redistribution might indicate that axoneme shortening is a consequence of the redistribution of the IFT machinery resulting in depletion of free tubulin at the axonemal tip. On longer time scales, the TBB4::GFP fluorescence distribution does not show as clearly a recovery as IFT dynein.

To visualize the distribution of TPs involved in chemosensation and their dynamics during avoidance behavior, we generated worms endogenously expressing the fluorescently labeled GPCR SRB-6 (SRB6::EGFP) and transmembrane calcium channel OCR-2 (OCR-2::EGFP) (Tobin et al., 2002; Tran et al., 2017). Expression levels of SRB-6::EGFP were low, only several tens of molecules could be observed in each phasmid cilia pair (Figure S2, Movie S2). Such expression levels are too low to perform repellent experiments, which require long-term imaging without photobleaching. High laser powers are required to visualize such a small number of molecules, resulting in bleaching of all present molecules within seconds. Expression levels of OCR-2, on the other hand, were much higher, making it possible to image OCR-2 long enough at laser powers low enough to avoid substantial photobleaching during the course of the experiment.

To probe whether the redistribution of IFT dynein and the partial shortening of the axoneme caused by SDS has an effect on the distribution of TPs like OCR-2, we performed SDS-addition experiments with worms expressing OCR-2::EGFP. Time-averaged fluorescence images show that, before application of 
SDS, OCR-2 is distributed unevenly over the cilium (Fig 1A): it appears to accumulate at the base, in the DS and the tip, while it is less abundant in the middle of the cilium and almost absent in the TZ (see also below). After SDS addition, OCR-2 redistributes, becoming less abundant in tip and DS, but to a different extend than IFT dynein and tubulin. Kymographs showing the OCR-2::EGFP distribution confirm the observation that OCR-2 leaves the DS (Figure 1B). Part of the OCR-2 remains, indicating that the ciliary membrane does not retract from the DS (together with the microtubules). For further quantification of the changes in fluorescence intensity, we divided the cilia in four segments, as indicated on top of the kymograph (including the ciliary tip as distinct segment). The fluorescence intensity time traces in these four different segments show that, while the amount of OCR-2 in the PS remains more or less constant, approximately one third of the proteins in the tip disappears, and an equal amount reappears in the DS and PCMC (Figure 1C). Together, these data show that the cilium is capable of rapid redistribution and recovery of IFT in the distal segment, resulting in a partial shortening of the axoneme and a reduction in the amount of OCR-2 in the tip region.

Rapid redistribution of IFT dynein and tubulin away from the DS is reminiscent of the laser ablation and azide dosing experiments we have performed before (Mijalkovic et al., 2020). Here it was shown that ablation of the phasmid chemosensory neurons at the dendrite (a couple of micrometers before the start of the cilium), resulted in the activation of retrograde IFT, leading to a redistribution of IFT components out of the DS towards the base on a time scale similar to the SDS-addition experiments presented here. ATP did not seem to be involved in the initial response, since ATP depletion by azide-addition did not lead to the activation of retrograde IFT. Instead, upon depletion of ATP, IFT components slowed down, redistributed towards the base and eventually left the cilium, comparable to the response to laser ablation at longer time scales. Together, these data were interpreted to reveal an IFT-regulatory mechanism that allows rapid redistribution of ciliary components in response to changes in the outside environment. In the present SDS-addition experiments we see qualitatively similar redistributions of tubulin and IFT components, with one crucial difference: the redistribution starts about one minute after the SDS addition and almost concomitant avoidance response (within a few seconds), while redistribution starts within a few seconds after laser ablation or azide-addition. So, although immediate redistribution is possible in the cilium, it seems that upon SDS addition, the trigger for redistribution is delayed. Since the response time for second messengers is known to be within seconds, they are not likely the cause of the delay (Jiang et al., 2019; Pifferi et al., 2006; Tran et al., 2017). In our experiments, the worms are exposed to a prolonged SDS stimulus. This might indicate that the localization of sensory TPs affects cilium sensory sensitivity and that the redistribution effect observed here is a habituation response. 

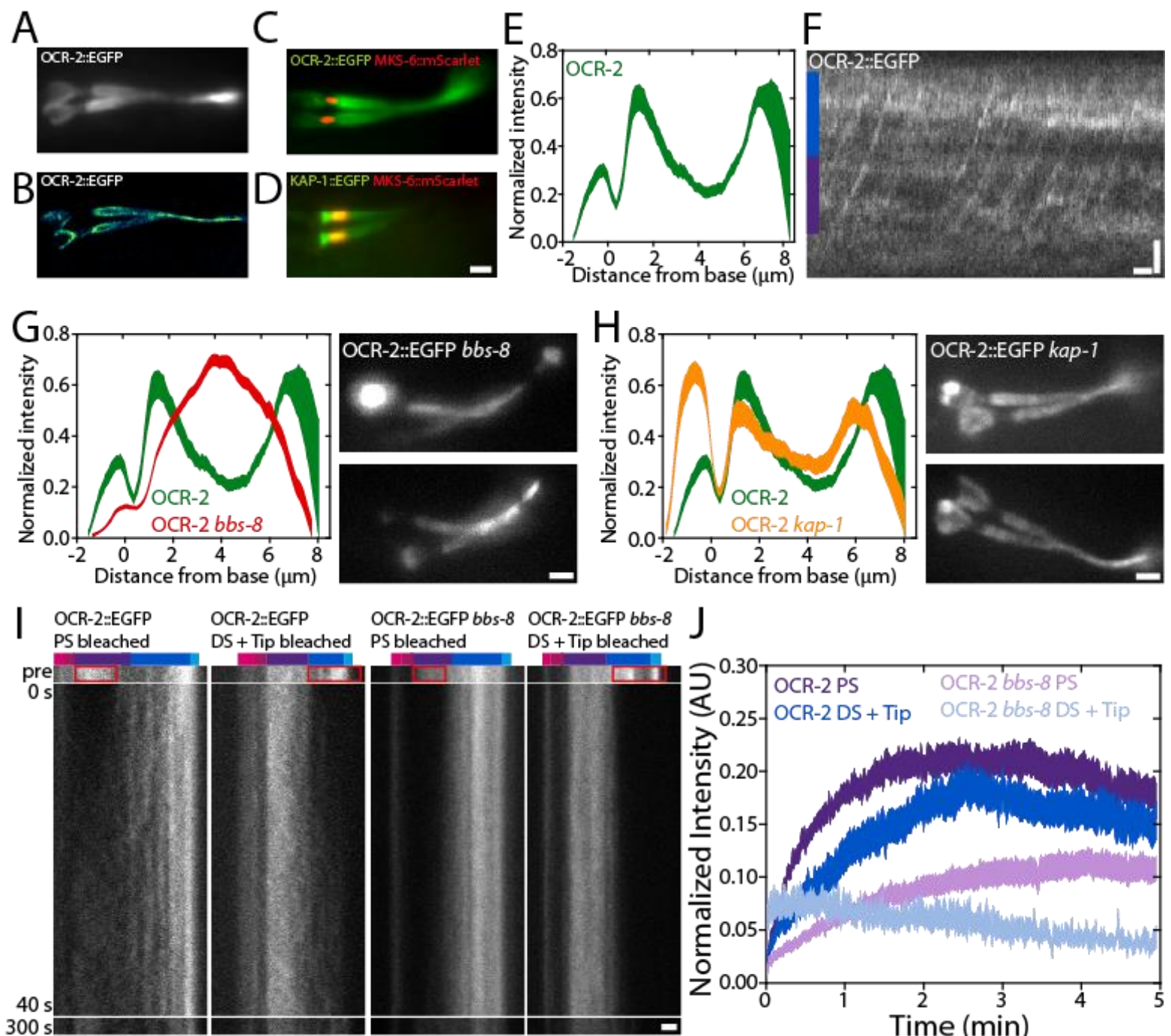

OCR-2:EGFP bbs-8 OCR-2=EGFP bbs-8

PS bleached DS + Tip bleached
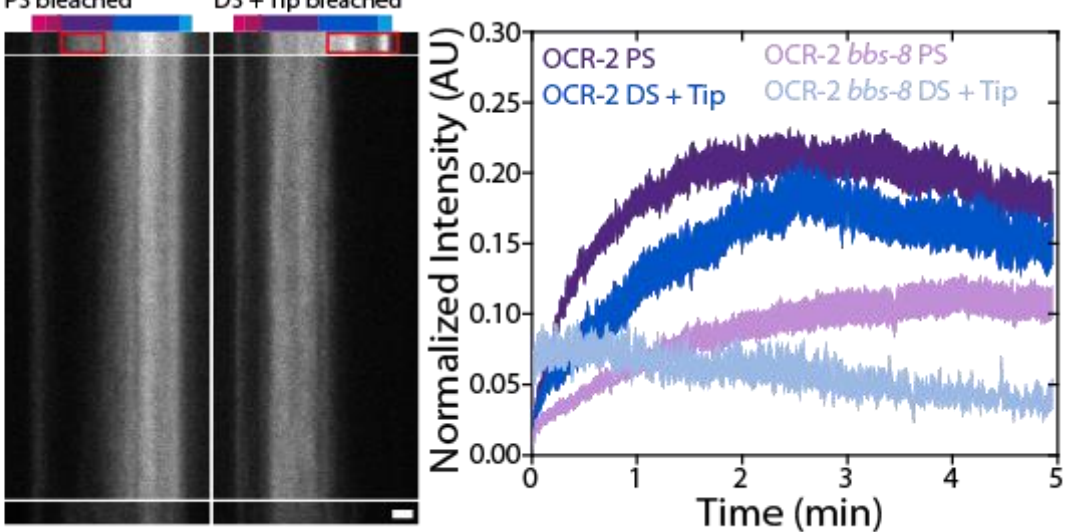

Figure 2: Ensemble distribution and dynamics of OCR-2. (A) Time-averaged (over 3 s) fluorescence images of OCR-2::EGFP. (B) Super-resolution image of OCR-2::EGFP molecule localizations. (C) Dualcolor image of endogenously labeled OCR-2::EGFP and overexpressed MKS-6::mScarlet. (D) Dual-color image of endogenously labeled Kinesin-II(KAP-1::EGFP) and MKS-6::mCherry. (E) Average intensity distribution of OCR-2::EGFP along the cilium ( $\mathrm{n}=22$ worms, normalized intensity distributions). (F) Kymograph of ensemble dynamics of OCR-2::EGFP with bars indicating the PS (purple) and DS (dark blue). Scale bars: $1 \mu \mathrm{m}$ and $1 \mathrm{~s}$. (G) Left: normalized average intensity distribution of OCR-2::EGFP (green) and OCR-2::EGFP bbs-8 (red) $(\mathrm{n}=71)$ along the cilium. Right: Time-averaged fluorescence images of OCR-2::EGFP bbs-8. (H) Left: normalized average ciliary distribution of OCR-2::EGFP (green; same data as E) and OCR-2::EGFP, kap-1 mutant (red) ( $\mathrm{n}=26$ worms). Right: Time-averaged (over $3 \mathrm{~s}$ ) fluorescence images of OCR-2::EGFP, kap-1 mutant. Scale bar: $1 \mu \mathrm{m}$. (I) Representative kymographs before and after photo bleaching the PS, or DS and Tip of OCR-2::EGFP and OCR-2::EGFP $b b s-8$. Colored bars indicate segments as in Figure 1B. Time scale is identical for all kymographs. (J) Normalized average intensity of OCR-2::EGFP in PS, or DS and Tip, after photobleaching PS or DS and Tip respectively, in WT and $b b s-8$ animals (OCR-2::EGFP PS: $\mathrm{n}=11$, DS = Tip: $\mathrm{n}=10$; OCR-2::EGFP bbs-8 PS: $\mathrm{n}=16$, DS + Tip: $\mathrm{n}=10$ ). 


\section{Ensemble distribution and dynamics of OCR-2}

We next had a closer look into the distribution of OCR-2 along the cilium. First, time-averaged fluorescence images were generated from image sequences of a $C$. elegans strain expressing OCR-2::EGFP (Figure 2A-D). In order to localize the TZ with respect to the OCR-2 distribution, we imaged phasmid cilia in worms overexpressing the TZ marker MKS-6::mScarlet and with endogenously labeled OCR-2 (Figure 2C-D). From such images, the intensity distribution along the cilium long axis was calculated (Figure 2E). The images and intensity distribution indicate that the $\mathrm{TZ}$ corresponds to a region with very low OCR-2 density. Anterior to the TZ, OCR-2 appears to accumulate in a cup-like shape, which most likely corresponds to the PCMC. Posterior to the TZ, the intensity of OCR-2 is relatively high, dropping further along the middle segment and increasing again in the DS, peaking at the ciliary tip. A superresolution image obtained from many localizations of individual OCR-2 molecules (see below and Oswald et al., 2018), confirms these observations (Figure 2B), highlighting the membrane localization of OCR-2, in particular in the wide PS.

To get a first insight in what underlies the distribution of OCR-2 along the cilium, we generated kymographs from the original image sequences at the ensemble level (Figure 2F). The kymograph clearly shows directional, processive movement of OCR-2, in agreement with previous studies of worms expressing fluorescent OCR-2 from extra-chromosomal arrays (Qin et al. 2005). The continuous lines due to motor-driven OCR-2, however, are less clear than what we have observed before for motor proteins and other IFT components (Mijalkovic et al., 2017; Prevo et al., 2015) and appear to be obscured by a relatively high, unstructured background signal of OCR-2. This might indicate that OCR-2 is only partly actively transported by IFT and to a substantial account by passive diffusion.

In order to determine how essential active transport by IFT is for the ciliary distribution of OCR-2, we generated worms lacking BBS-8, which is crucial for a functional BBSome, and thereby for the connection between ciliary membrane proteins and the IFT machinery (Blacque et al., 2004). Kymographs generated from image sequences, indeed, do not show any signs of active transport of fluorescent OCR-2 in the absence of BBS-8 function, but do show that IFT is still active (Figure S3). Fluorescence images were time averaged in order to generate an intensity distribution averaged over multiple cilia (Figure 2G). These images show that OCR-2 does enter the cilia of $b b s-8$ worms, but that its distribution along the cilium is remarkably different compared to wild-type animals: OCR-2 concentration does not peak behind the TZ and at the tip (as found in wild type), but shows a maximum in the middle of the cilium. We note that the time-averaged fluorescence images of $b b s-8$ worms were quite heterogeneous. In some worms, substantial accumulations of OCR-2 were observed in the PCMC (e.g. bottom image in Figure 2G) suggesting that import of OCR-2 is severely hampered in these cilia. Together, these results show that active transport plays an important role in the ciliary distribution of the TP OCR-2. Furthermore, although a functional BBSome has been shown to be necessary for avoidance behavior and is involved in ciliary exit of TPs (Blacque et al., 2004; Lechtreck et al., 2013), it seems not completely essential for ciliary entry of OCR-2 into the cilium. Together, this might indicate that the proper, IFT-dependent, ciliary distribution of TPs involved in chemotaxis is required for the propagation of environmental stimuli.

To study the effect of a more subtle modulation of active transport of TPs, we generated worms with endogenously labeled OCR-2, but lacking Kinesin-II function (tm123). Kinesin-II drives, together with OSM-3, anterograde IFT, with Kinesin-II mostly active from the ciliary base across the TZ and OSM-3 in 
the rest of the cilium (Prevo et al., 2015). While mutant worms lacking OSM-3 function have short cilia lacking a DS and are osmotic avoidance defective, worms lacking Kinesin-II function do show osmotic avoidance behavior and exhibit only minor structural defects (Oswald et al., 2018; Snow et al., 2004). Time-averaged image sequences and the average intensity distribution of OCR-2 in kap-1 mutant cilia show accumulations of OCR-2 posterior to the TZ and at the ciliary tip, just as wild type, but also show a far more pronounced accumulation in the PCMC (Fig 2J). These observations are in line with earlier observations of the distributions of IFT-particle components in kap-1 mutant strains, which showed similar distributions along the cilium compared to wild type, but also showed accumulations in the PCMC, suggesting that Kinesin-II is a more efficient import motor than OSM-3 (Prevo et al., 2015). Furthermore, these observations are in line with those of OCR- 2 in bbs- 8 mutant worms, supporting the conclusion that efficient, active transport of OCR-2 by IFT is essential for its distribution and function.

To obtain insight in the ensemble dynamics that underlie the ciliary distribution of OCR-2, we performed Fluorescence Recovery After Photobleaching (FRAP) experiments on OCR-2::EGFP in wild-type and bbs-8 mutant worms (Fig 2I), photobleaching the EGFP in PS, or DS and tip, by brief, intense laser illumination. Image sequences of the fluorescence recovery were converted into kymographs of the cilium (Fig 2I) and fluorescence intensity time traces integrated over the ciliary segments (Fig 2J). Kymographs and time traces show that, in wild-type cilia, the rate and extend of OCR-2::EGFP recovery in the PS after bleaching the PS, is slightly different from the recovery in DS and Tip after bleaching DS and Tip. In the PS, only $-21 \%$ of the intensity before bleaching the PS recovered after -2 minutes, while in the DS and Tip $-18 \%$ recovered -2 minutes after bleaching DS and Tip. This indicates that in the cilium, only a small fraction of OCR-2 is free to move, driven by IFT or free diffusion.

In bbs- 8 mutant worms (where the connection between OCR-2 and IFT machinery is disrupted), the mobile fraction is even smaller in the PS $(-11 \%)$ and DS $(-5 \%)$ compared to those segments wild type. Compared to wild type, a similar difference in the recovery rate and extend between the PS and DS in the bbs- 8 mutant cilia was observed. The difference of the mobile fractions in the PS and DS between wild type and $b b s-8$ mutant worms furthermore demonstrates the importance of IFT for the ciliary distribution of OCR-2. This difference is larger in the DS, suggesting IFT plays a larger role in the DS for OCR-2 localization.

Collectively, these findings demonstrate the diversity of transport modalities that underlie the ensemble dynamics resulting in the steady-state distribution of OCR-2 along the cilium. This distribution is not uniform along the cilium but shows accumulations at the Tip and posterior to the TZ, and a smaller accumulation anterior to the TZ. In mutant animals with perturbed active transport of OCR-2, the distribution is different. In addition, our imaging and FRAP analysis indicate that diffusion of OCR-2 in the ciliary membrane also plays a key role. 


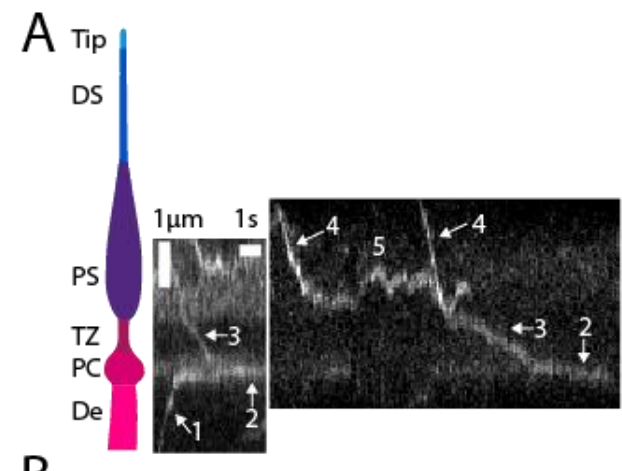

KAP-1
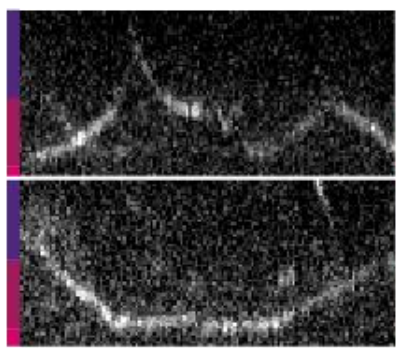
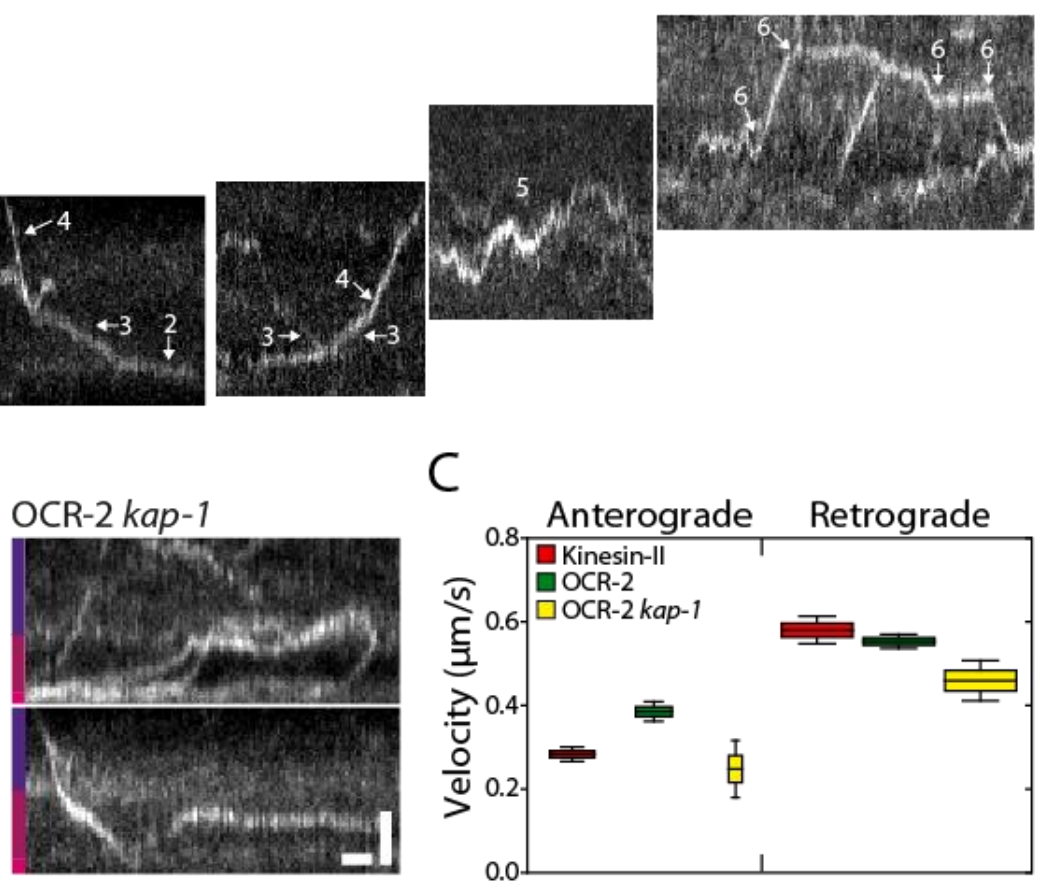

Figure 3: Single-molecule imaging of OCR-2. (A) Cartoon of the ciliary segments and representative single-molecule kymographs of OCR-2::EGFP. Scale bars, vertical: $1 \mu \mathrm{m}$, horizontal: 1 s. (B) Singlemolecule kymographs showing TZ crossings of Kinesin-II (KAP-1::EGFP) and OCR-2::EGFP kap1(tm123). Color bars indicate ciliary segment as in (A). Scale bars, vertical: $1 \mu \mathrm{m}$; horizontal: $1 \mathrm{~s} .(\mathrm{C})$ Anterograde (A) and retrograde (R) velocity of KAP-1::EGFP (A: 63 trajectories; R: 273 trajectories), OCR-2::EGFP (A: 51 trajectories; R: 297 trajectories) and OCR-2::EGFP kap-1(tm123) (A: 19 trajectories; R 141 trajectories) (error bars represent s.e.m.).

\section{Single-molecule imaging reveals large diversity in OCR-2 motility}

In order to obtain more detailed insight into the extents by which diffusion and active transport drive OCR-2 motility along the cilium, we performed single-molecule tracking of OCR-2::EGFP in live $C$. elegans using laser-illuminated wide-field epifluorescence microscopy, at a frame rate of $10 \mathrm{~Hz}$. In Figure 3A (see also Movie S3), example trajectories are represented as kymographs, with location in the cilium indicated. The kymographs suggest a variety of different behaviors of OCR-2, ranging from diffusion to directed transport in anterograde and retrograde directions. The specific behavior appears to depend on the actual location in the cilium. In the dendrite, straight, slanted lines are observed towards the PCMC (see also Figure S4) (1), indicative of active, directional transport of OCR-2. In the PCMC, kymograph lines appear mostly horizontal but slightly wavy (2), indicative of particles lingering a substantial amount of time (which appears to be limited by photobleaching in our experiments). In the TZ, again straight, slanted lines are observed (3), indicating that particles cross the TZ mostly by directed transport. In the PS, more heterogeneous behavior is observed. Some particles show straight, slanted lines (4), indicative of active transport, while others appear saltatory (5), indicating that particles diffuse or move by short bouts of active transport in anterograde or retrograde direction. In the DS, a similar pattern of directed motion in combination with saltatory movement is observed (6), albeit that the fraction of directed transport appears higher. Taken together, single-molecule kymographs hint at a large diversity of OCR-2-motility patterns that appear to depend on the specific ciliary segment and location. 
One of the observations in the kymographs of Fig 3A is that the motion of OCR-2 in the TZ appears to be mostly directional, driven by IFT. The motility of cargo OCR-2 appears very similar to that of motor Kinesin-II (imaged using a KAP-1::EGFP strain; Figure 3B), also with comparable velocity in anterograde and retrograde direction (Figure 3C). These observations strongly suggest that OCR-2 is indeed transported across the TZ by IFT trains driven by Kinesin-II in the anterograde direction and IFT dynein in the retrograde direction. The densely packed protein network (e.g. the Y-shaped linkers connecting axoneme and membrane) of the TZ forms a diffusion barrier between dendrite and PS (Garcia-Gonzalo and Reiter, 2017). We have shown before that in the absence of Kinesin-II function (in the kap-1 mutant strain), OSM-3 can take over and drive IFT across the TZ. In these strains, however, the frequency and number of IFT trains entering the PS appears affected and IFT components show more substantial accumulations in front of the TZ (Prevo et al., 2015). In line with these observations, we have shown above that OCR-2 is still present in cilia lacking kinesin-II function, but that its ciliary distribution is affected (Figure 2H). Single-molecule kymographs generated from image sequences of kap-1 mutant worms show many more OCR-2 molecules being stuck in the TZ (Figure 3B), or traversing it more irregularly than in wild-type animals, although successful $\mathrm{TZ}$ crossings can still be observed. Velocities extracted from the trajectories were only slightly lower than for wild type (Figure 3C), which might seem remarkable, since OSM-3, which is generally considered to be a faster motor, drives anterograde crossing (in the kap-1 mutant) instead of Kinesin-II (in wild type). We have, however, shown before that OSM-3 velocity in the TZ of kap-1 mutant worms is substantially reduced in addition to affecting OSM-3's efficiency to traverse the $\mathrm{TZ}$, most likely due to $\mathrm{TZ}$ structures acting as roadblocks for OSM-3, more severely than for Kinesin-II (Prevo et al., 2015). Taken together, the TZ acts as an efficient diffusion barrier for OCR-2, which needs to be overcome by active transport driven by IFT. In mutant worms lacking Kinesin-II function, OCR-2 transport over the TZ can be taken over by OSM-3, albeit with loss of efficiency. In both wild-type and kap-1 mutant worms, IFT dynein drives effective retrograde transport of OCR-2.

A

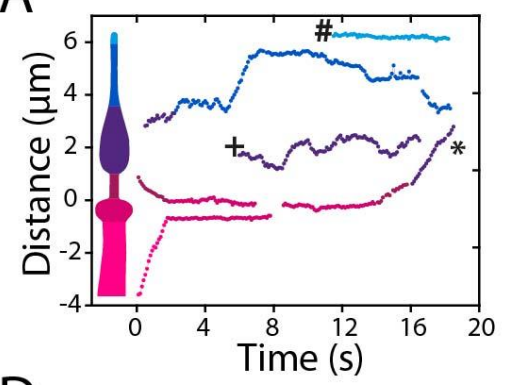

D

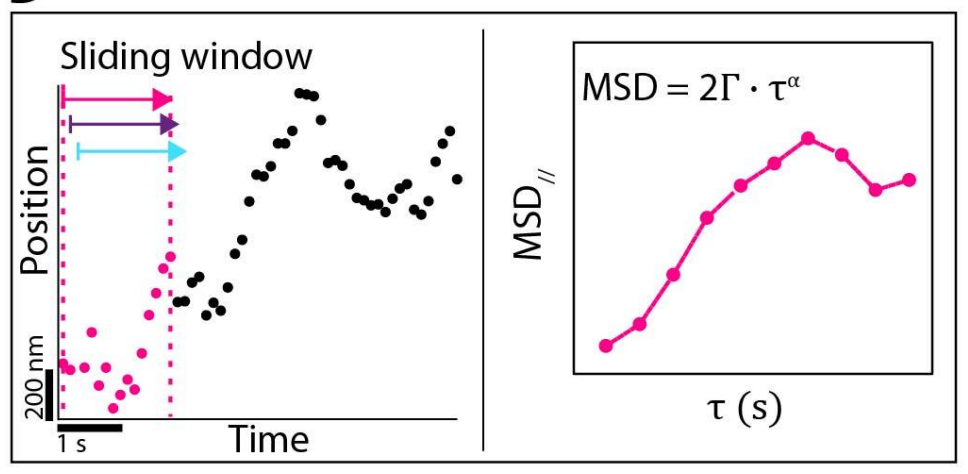

B

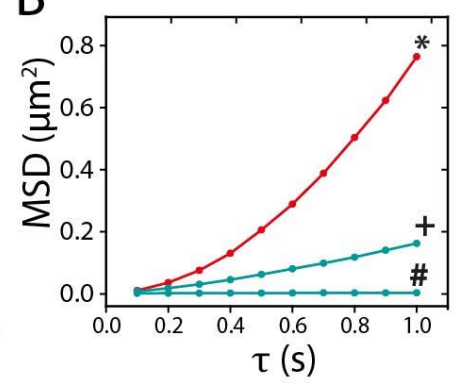

E

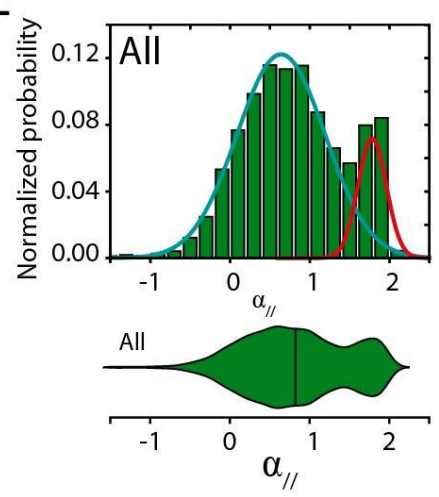


Figure 4: Analysis of single-molecule OCR-2 trajectories. (A) Example single-particle trajectories from the kymographs of figure 3A. (B). Mean squared displacement (MSD) of annotated trajectories in (A), demonstrating active transport $(*)$, normal diffusion $(+)$ and subdiffusion $(\#) .(\mathrm{C})$ Log-log plot of the first six time points. (D) Cartoon explaining the single-molecule data analysis with sliding window. (E) Histogram and corresponding violin plot of normalized probability of $\alpha_{/ /}$for all 6992 data points obtained from 23 nematodes. Colored lines represent two underlying Gaussian distributions.

Single-molecule imaging reveals bimodal motility distribution of ciliary OCR-2

350

351

352

353

354

355

356

357

358

359

360

361

362

363

364

365

366

367

368

369

370

371

372

373

374

375

376

377

378

379

380

381

382

383

To get a more quantitative insight in the motility behavior of single OCR-2 molecules inside the entire cilium, we tracked single molecules in images sequences, such as those represented in the kymographs of Figure 3A, using a local ciliary coordinate system (parallel and perpendicular to a spline drawn along the long axis of each cilium). Representative trajectories are shown in Figure 4A, with examples of a trajectory suggestive of active, directed transport $\left({ }^{*}\right)$, a trajectory suggestive of diffusive transport $(+)$ and a trajectory of a molecule that hardly moves in the ciliary tip (\#). For further analysis, we calculated the mean squared displacement (MSD) of these example trajectories (Figure 4B\&C). MSD analysis is a widely used approach to characterize motility, based on the scaling of MSD with time (Gal et al., 2013). This scaling can be expressed in one dimension as: $M S D(\tau)=2 \cdot \Gamma \cdot \tau^{\alpha}$, with, $\Gamma$ the generalized transport coefficient, $\tau$ the time lag, and $\alpha$ the exponent. In case $\alpha=2$, the motion is purely directed (ballistic) and the velocity $(v)$ is equal to $\sqrt{2 \Gamma}$. When $\alpha=1$, the motion is due to normal diffusion and characterized by a diffusion coefficient $(D)$ equal to $\Gamma$. In case $\alpha<1$, the motion is considered subdiffusive (MacKintosh, 2012), which in this case could be caused by association of OCR-2 to stationary structures and / or by the restriction of OCR-2 motion due to other, slower moving proteins (Saxton, 1994). Application of this MSD analysis to the three example trajectories confirms the different modes of motility underlying these trajectories: the MSD of the apparently directed trajectory $\left({ }^{*}\right)$ scales roughly quadratically with time, that of the diffusive trajectory $(+)$ linearly, and that of the hardly moving molecule (\#) with a slope less than linear, indicative of subdiffusion.

The analysis used above was applied to user-selected (fragments of) trajectories with different length. In order to obtain unbiased insight from all 185 trajectories (in 23 worms) constituting 6992 individual localizations, we calculated the instantaneous MSD, using a sliding window of 15 consecutive locations, in accordance with previous work (Godin et al., 2017), yielding instantaneous values of $\Gamma$ and $\alpha$, both parallel and perpendicular to the cilium (Figure 4D and methods) (Danné, 2018). As a first characterization of the data obtained in this way, we plotted all $\alpha / /$ values in a histogram and corresponding violin plot (Fig 4E). Histogram and violin plot clearly show a bimodal distribution of $\alpha_{/ /}$ confirming that OCR-2 moves by a combination of directed and diffusive motion. A fit with two Gaussians to the histograms yields a diffusive fraction (84\%) with average $\alpha_{/ /}$of 0.66 (Full Width Half Maximum (FWHM): 0.56), and a directed motion fraction (16\%) with average $\alpha_{/ /}$of 1.78 (FWHM: 0.17). This indicates that the majority of motion of OCR-2 in the cilia is diffusive. Furthermore, in particular the diffusive fraction is broadly distributed, indicating heterogeneous diffusive behavior. In the remainder of this study we will use $\alpha=1.4$, the intersection between the two Gaussians fits, as the threshold to discriminate between diffusive and directed motion. This analysis indicates that instantaneous values of $\alpha$ can be readily obtained from single-molecule trajectories of OCR-2 in cilia and can provide quantitative insight in OCR-2 dynamics. 


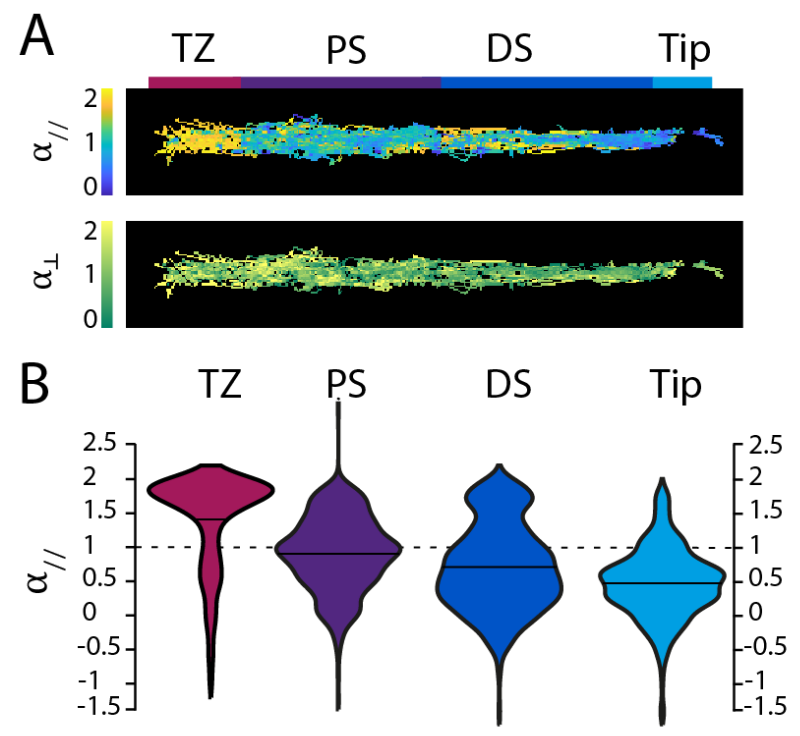

C
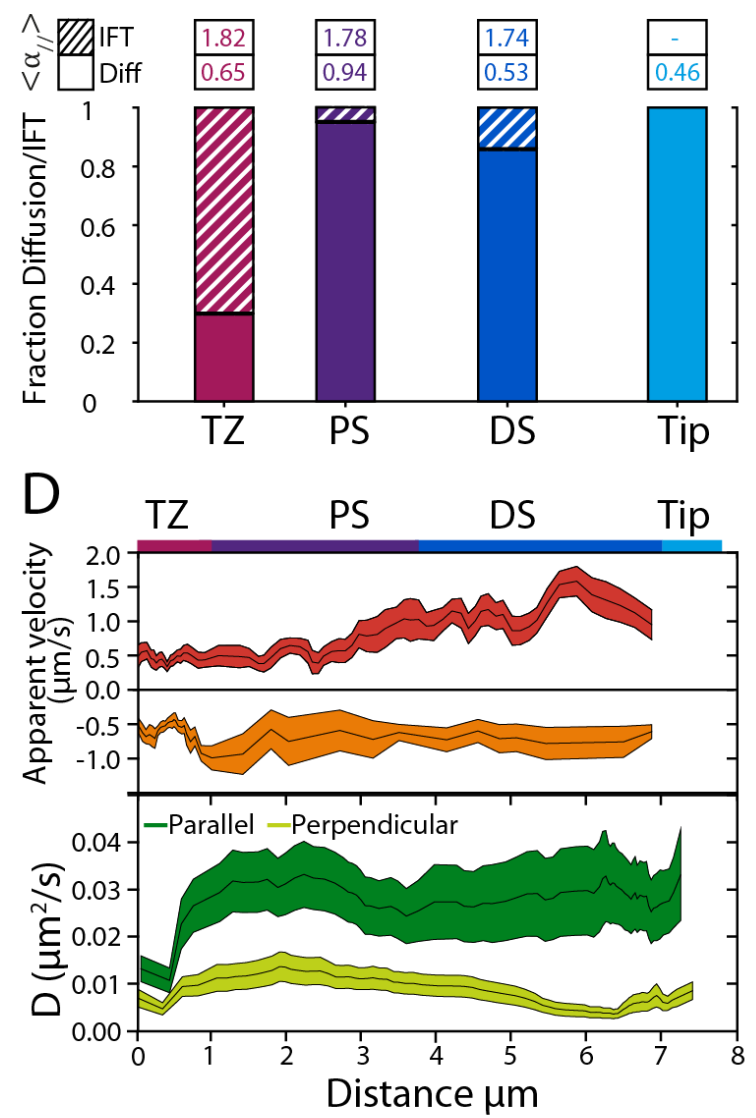

Figure 5: Analysis of single-molecule OCR-2 trajectories reveals location-specific motility. (A) Heat maps showing all data points color coded for $\alpha_{/ /}$and $\alpha_{\perp}$. Scale bar: $1 \mu \mathrm{m}$. (B) Violin plots showing $\alpha_{/ /}$ for the TZ, PS, DS and Tip regions of the C. elegans phasmid cilia. (C) Relative fractions of molecules undergoing IFT or diffusive motion, with corresponding average $\alpha_{/ /}$in the different ciliary segments. (D) Apparent velocities and diffusion coefficients of OCR-2 molecules in the phasmid cilia. Top: apparent anterograde and retrograde velocities determined for time points for which $\alpha_{/ /} \geq 1.4$, averaged over 10 displacements. Line thickness represents s.e.m.. Bottom: parallel and perpendicular diffusion coefficients 
of OCR-2 determined for time points for which $0.9 \leq \alpha_{/ /, \perp} \leq 1.1$, averaged locally over 60 displacements. Line thickness represents s.e.m..

\section{A location-specific interplay of transport dynamics underlies the ciliary distribution of OCR-2}

Next, we used the same dataset to obtain more quantitative insight into the motion of OCR-2 in the different ciliary segments. To obtain an overview of the location-specific motility properties of OCR-2, all $\alpha_{/ /, \perp}$ and $D_{/ /}$values, derived from the instantaneous MSD, were color coded for $\alpha$ and plotted in function of their location (Fig 5A). Even though the data used for these heat maps is derived from 23 different animals, the resulting shape resembles the shape of a single cilium, validating the robustness of our imaging and analysis. This representation furthermore highlights that OCR-2 motility parameters depend on the location in the cilium. To further quantify this, we represented the distributions of the $\alpha_{/ /}$ values in the different ciliary segments in violin plots (Figure 5B). The violin plots show that the distributions vary substantially between the segments. In the TZ, $\alpha_{/ /}$values peak close to two; in the PS $\alpha_{/ /}$values are widely distributed, peaking around 1 ; in the DS, two peaks can be observed, the major, broad one peaking around 0.5 and the minor one peaking slightly below 2; in the Tip, a single distribution around 0.5 can be seen. The results of Gaussian fits to these distributions (Figure S5) is represented in Figure 5C, which shows the fractional contributions for directed motion and diffusion in the different ciliary compartments and the corresponding average $\alpha_{/ /}$values. Taken together, Figure 5B and $\mathrm{C}$ confirm, in a quantitative way, the initial indications obtained from the kymographs. In the TZ, OCR-2 moves mostly by directed transport; in the PS, OCR-2 moves mostly by normal diffusion in the membrane and only to a small extend via directed transport; in the DS, the fraction of OCR-2 moving by directed transport is slightly higher, while the rest moves subdiffusively; at the tip all motion is subdiffusive.

We further analyzed our data by determining the apparent local velocities from (stretches of the) trajectories with $\alpha_{/ /} \geq 1.4$, using $v_{a p p}=(x(t+d t)-x(t)) / d t$, with $d t$ the frame integration time (Holcman et al., 2018), averaging locally over 10 displacements (Figure 5D). Local apparent anterograde velocity compares very well to velocity profiles obtained before using bulk (Prevo et al., 2015) and singlemolecule fluorescence imaging (Oswald et al., 2018) of kinesin motors and other IFT components: with a velocity of about $0.5 \mu \mathrm{m} / \mathrm{s}$ close to the TZ, gradually increasing in PS, reaching the maximal value of more than $1 \mu \mathrm{m} / \mathrm{s}$. This similarity of the anterograde OCR-2 velocity profiles presented here, compared to those obtained from the IFT components reported before, highlights the validity of our approach. Apparent retrograde velocities, however, agree less well with velocities reported before and are lower, in particular in the DS. We do not have a definitive explanation for this, but we note that the number of events showing directed minus end motion is substantially lower than for anterograde, potentially indicating that bouts of retrograde transport of OCR-2 are only short lived (compared to our sliding window of 15 frames, corresponding to $1.5 \mathrm{~s}$ ). In agreement with this, the OCR-2 kymographs show only few straight retrograde events in contrast to anterograde events, which are far more evident.

We next looked into the values of the apparent diffusion coefficients parallel and perpendicular to the ciliary axis $\left(D_{/ /, \perp}\right)$, calculated from (segments of) trajectories with $\alpha_{/ /, \perp} \leq 1.1$, using $D_{/ /, \perp}=$ 


$$
\left.\left(r_{/ /, \perp}(t+d t)-r_{/ /, \perp}(t)\right)^{2} / d t \text { (with } r_{/ /, \perp}=x, y\right) \text { (Holcman et al., 2018), averaging locally over } 60
$$
actively transported by IFT by adding as constraint that only time windows were included with $\alpha_{/ /}<1.4$, in order to allow for a proper comparison of the diffusion coefficients in both directions. Using this approach, we found that $D_{/ /}$was rather constant, $-0.03 \mu \mathrm{m}^{2} / \mathrm{s}$, along the cilium, except for the TZ, where it was $\sim 0.01 \mu \mathrm{m}^{2} / \mathrm{s}$. $D_{\perp}$ was almost three times smaller than $D_{/ /}-0.01 \mu \mathrm{m}^{2} / \mathrm{s}$, decreasing somewhat in the distal segment. In part, the lower $D_{\perp}$ compared to $D_{/ /}$could be a consequence of our imaging approach, which effectively results in a 2-dimensional projection of a 3-dimensional object, making us blind for motion in the direction perpendicular to the image plane (which is one of the two axes perpendicular to the cilium). This could result in an underestimation of the diffusion coefficient in the direction perpendicular to the cilium, at most by only a factor of about 2 (Renner et al., 2011; Van Den Wildenberg et al., 2011). We speculate that the larger difference between the apparent $D_{/ /}$and $D_{\perp}$ in DS and Tip might be caused by confinement, which could result in subdiffusive motion.

444

Taken together, single-molecule imaging reveals the extent of the location-specific diversity in motility modes of OCR-2 in C. elegans chemosensory cilia, ranging from active transport, normal diffusion, to subdiffusion. This combination of motility modes is crucial for establishing the steady-state distribution of OCR-2 over the cilium, which is essential for effective OCR-2 function, allowing the binding of aversive chemicals to receptors to provoke an avoidance reaction of the whole animal.

\section{Discussion}

Exposed to repellent chemicals, C. elegans performs avoidance behavior. In this study, we have aimed to connect this whole-organism behavior to the dynamics of the molecular machinery inside the chemosensory cilia that sense the chemicals and trigger the subsequent processes that drive behavior. To this end, we have exposed C. elegans to aversive chemicals and imaged the distribution and dynamics of ciliary components using fluorescence microscopy. Our image sequences show that exposure to SDS results in a rapid ( $-10-20 s)$, reversible redistribution of tubulin, IFT-dynein, and OCR-2 out of the DS, about a minute after exposure to SDS. Furthermore, with single-molecule imaging, we have shown that the transmembrane calcium channel OCR-2 displays complex location-specific motility, ranging from subdiffusion to active transport by IFT. Together, these diverse motility modes help to establish OCR-2 distribution along the cilium and allow for its reversible redistribution.

Whereas the SDS-evoked avoidance response occurs almost instantaneously after SDS addition, the redistribution of ciliary components starts much later, about a minute after addition, suggesting that the redistribution is not an intrinsic part of the avoidance response. Laser-ablation experiments of the $C$. elegans phasmid dendrite and live imaging of IFT and calcium in Chlamydomonas have shown that the distribution of IFT components and IFT dynamics can be altered almost instantaneously (Collingridge et al., 2013; Mijalkovic et al., 2020). The cause of the delay in our SDS-addition experiments is hence not the inability of the cilia or IFT to react rapidly. Since in our experiments SDS exposure was prolonged, it is well possible that the delayed redistribution is part of a sensory habituation mechanism. Sensory habituation has been studied before in the ASH amphid chemosensory neurons in C. elegans (Hilliard et al., 2005). These experiments revealed that the calcium concentration in the ASH somas (and as a consequence the neuronal response) decreased on a -minute time scale during prolonged repellent 
exposure (Hilliard et al., 2005). The calcium response to repellents was also shown to decrease after short, consecutive stimuli. Taken together, this suggests that the reversible redistribution of ciliary components we observed a -minute after SDS exposure is indeed part of a sensory habituation mechanism, distinct from the avoidance response. Although questions remain about the exact function of the redistribution of ciliary components in chemosensing, it is well possible that the plasticity of the axoneme and ciliary composition could be involved in a mechanism increasing the dynamic range of chemosensing in order to minimize (prolonged) exposure to potentially dangerous compounds.

Distribution and motility of TPs involved in sensing have been studied in the cilia of other model systems, in particular in primary cilia of cultured mammalian cells (Huang et al., 2007; Milenkovic et al., 2015; Qin et al., 2005; Weiss et al., 2019; Ye et al., 2013). The advantage of using a multicellular organism such as C. elegans as a model organism is that, in principle, the whole cascade from sensory membrane protein, to secondary messengers, neuronal action and finally behavior can be studied in the same model. The price one has to pay is that high-sensitivity imaging is hampered by signal loss due to scattering and optical aberrations caused by imaging relatively deep into the tissue of the animal. Using our approach, it works well for the phasmid cilia in the tail of the nematode, but not for the amphid cilia in the head, because of more out-of-focus background fluorescence, higher autofluorescence of the surrounding tissue and substantially more movement of the animal. Imaging primary cilia of mammalian cultured cells poses, in this regard, substantially less challenges.

It is important to note that, although $C$. elegans chemosensory cilia are highly homologous to mammalian primary cilia, important differences exist. Whereas the primary cilia of cultured mammalian cells are slightly shorter and consist of an axoneme with doublet microtubules along almost their entire length, $C$. elegans cilia consist of an axoneme with doublet microtubules only until about halfway, with microtubule singlets extending towards the ciliary tip in the DS. Furthermore, C. elegans chemosensory cilia are located inside the amphid and phasmid channels with only the tip sticking out into the environment, while mammalian cilia are completely exposed. This might explain why OCR-2 in C. elegans chemosensory cilia accumulates at the tip, whilst GPCRs like Somatostatin Receptor 3 (SSTR3) and Smoothened, in mammalian primary cilia are more evenly distributed along the length of the cilia (Milenkovic et al., 2015; Ye et al., 2013).

Despite these fundamental differences in ciliary properties, our findings on the motility of OCR-2 in $C$. elegans phasmid cilia qualitatively agree with those of TPs in primary cilia of cultured mammalian cells. In C. elegans we found that OCR-2 motility is mostly due to diffusion and only less than $20 \%$ by active transport, IFT. Also in mammalian primary cilia, GPCRs have been found to be mostly diffusing, and only partly moved by IFT ( $-32-34 \%$ (Ye et al., 2013) and $\sim 1 \%$ (Milenkovic et al., 2015) IFT for Smoothened; $-13-27 \%$ for SSTR3 (Ye et al., 2013); -4\% for Patched1 (Weiss et al., 2019)). Remarkably, diffusion coefficients obtained for mammalian cilia are substantially larger (SSTR3: $-0.25 \mu \mathrm{m}^{2} / \mathrm{s}$ using FRAP (Ye et al., 2013); Smoothened $-0.26 \mu \mathrm{m}^{2} / \mathrm{s}$, using single-molecule tracking (Milenkovic et al., 2015); PTCH1: $\sim 0.1 \mu \mathrm{m}^{2} / \mathrm{s}$, PTCH1 using single-molecule tracking (Weiss et al., 2019)) than what we observed in $C$. elegans phasmid cilia $\left(-0.03 \mu \mathrm{m}^{2} / \mathrm{s}\right)$. It is unclear whether these are actual differences or caused by the differences in measurement and analysis approaches, some being better in discriminating active transport from diffusion. On the other hand, a lower diffusion coefficient could be caused by a larger size of the transmembrane section of the calcium channel OCR-2 compared to the mammalian 
GPCRs, or by species-specific differences in ciliary membrane viscosity, potentially caused by protein crowding (Oswald et al., 2016). Notwithstanding these qualitative differences, our results and those in mammalian primary cilia indicate that the combination of diffusive and active transport motility modes of TPs, and their ability to connect and disconnect to underlying IFT trains, is a conserved mechanism for the motility of TPs in cilia. More than in these previous studies on mammalian primary cilia, our studies in C. elegans phasmid cilia, which are longer and have structurally different domains, have revealed that TP motility in cilia is segment dependent: in the TZ, OCR-2 is mostly transported directionally by IFT; in the PS, normal diffusion is prevalent; in the DS, subdiffusion is most common, with noticeably more directed transport than in the PS; in the tip, OCR-2 motility is rather low, mostly subdiffusive. These location-dependent motility modes and parameters most likely underlie the steady-state distribution of OCR-2 along the phasmid cilia.

What would be the molecular basis for this location-dependent motility? Most likely there are two distinct processes underlying this behavior. The first process is a location-dependent connection of OCR-2 with the IFT machinery. The interaction of OCR-2 and other TPs to IFT trains has been shown to be mediated by the BBSome protein complex, which is essential for chemotaxis (Blacque et al., 2004). Recent cryo-EM studies of the BBSome have revealed molecular details of this interaction, a negatively charged cleft on the BBSome surface that is likely involved in the binding of cargo proteins (Klink et al., 2019). In another study it has been suggested that the GTPase ARL6/BBS-3 is activated when it interacts specifically with the BBSome, allowing for the connection between IFT-trains and transmembrane cargoes (Chou et al., 2019). This is in agreement with our results, which suggest that this interaction is tightly regulated, being persistent in the TZ, and far more intermittent in the rest of the cilium. This transient connection between IFT machinery and OCR-2 might explain the differences in ensemble and SM kymographs we have observed. Ensemble kymographs hinted at OCR-2 undergoing IFT from base to tip, while singlemolecule kymographs showed far less regular, more saltatory motion. The ensemble IFT trajectories most likely are the result of multiple OCR-2 independently attaching to and detaching from a particular IFT train. Further studies will be required to better understand the interaction of TPs and the IFT machinery and how this is locally regulated.

The second process underlying the location-dependent motility is the location-dependent (sub)diffusion. We have shown that OCR-2, when not connected to the IFT machinery, diffuses normally (with $\alpha \approx 1$ ) in the PS, while it moves subdiffusively in the DS and Tip (with $\alpha \ll 1$ ). Most likely, subdiffusion in the parallel direction is caused by an increased density of OCR-2 or other TPs (crowding), and/or static structural proteins, all hampering the motion of OCR-2 in the membrane. In addition, differences in membrane composition and curvature could also play a role, especially in the perpendicular direction in regions where the average distance explored during subdiffusion is close to the local diameter of the cilium. To further elucidate the molecular mechanisms inside the cilia underlying avoidance behavior, experiments will be required with a higher level of control over behavioral cues. In particular microfluidic devices could be employed to accurately control the exact instant and duration of a chemical stimulus (Chronis et al., 2007). This approach might allow imaging IFT at the single-molecule level, which is not feasible with our current approach. In addition, functional imaging approaches could be employed, including calcium probes to sense neuronal activity or probes that sense other secondary messengers including cAMP and cGMP (Imaging et al., 2019; Jiang et al., 2019). 
In conclusion, we have shown that sensitive fluorescence microscopy is an invaluable tool to study how whole-organism behavior is connected to single-protein dynamics. Investigating the molecular machinery underlying development and function of cilia provides important insights into mechanisms involved in key life processes such as sensing, signal transduction, limb patterning, and human disorders collectively named ciliopathies (Iii et al., 2018; Tobin and Beales, 2009).

\section{Materials and methods}

\section{C. elegans strains}

Strains used in this study were generated using MOSCI insertions and CRISPR/Cas9 genome editing and are listed in Table S1 (Frøkjær-Jensen et al., 2008; Paix et al., 2017). Primers and guide RNA's used to make the CRISPR/Cas9 and extrachromosomal array strains are listed in Table S2. Maintenance of $C$. elegans was performed using standard procedures (Brenner, 1974).

\section{Fluorescence microscopy}

Fluorescence microscopy was performed as described previously (van Krugten and Peterman, 2018). In short, C. elegans young adult hermaphrodites were immobilized using $5 \mathrm{mM}$ levamisole and mounted between a microscope slide with a $2 \%(\mathrm{~W} / \mathrm{V})$ agarose pad, and a coverslip. The worms were subsequently imaged on our custom-build wide-field epifluorescence microscope with $100 \mathrm{~ms}$ exposure time, and gently photo bleached to obtain single-molecule sensitivity.

\section{Repellent addition experiments}

Young adult hermaphrodite $C$. elegans were sedated as above, but mounted on a microscope slide with a central opening (AMIL Technologies). Imaging was performed as above. During the acquisition, $5 \mu \mathrm{l}$ $0.1 \%$ (W/V) sodium dodecyl sulfate (SDS) in M13 was pipetted through the central opening, which was then cover with a $22 \times 22 \mathrm{~mm}$ cover glass to prevent desiccation. The concentration of repellent at the worms was likely at least one order of magnitude lower than that what was added (Davies et al., 2003).

\section{Data analysis}

Kymographs were generated from the image sequences using the open source ImageJ plugin KymographClear and stand-alone program KymographDirect (Mangeol et al., 2016). Single-molecule tracking was performed as described previously, using custom-written MATLAB (MathWorks) routines using a linking algorithm (Jaqaman et al., 2008; Prevo et al., 2015). To be able to define and distinguish movement parallel and perpendicular to the ciliary axoneme, trajectory coordinates were transposed to a hand-drawn spline using the custom-written MATLAB script. In order to distinguish anterograde and retrograde velocities of OCR-2, we calculated the sign of the difference between two consecutives parallel positions $\operatorname{sign}(x(t+d t)-x(t))$. If the sign is positive, the directed motion is anterograde.

\section{Repellent experiments}

\section{Drift correction}

Drift coming from the movement of the worms was first corrected using the Fiji plugin TrackMate with the LoG (Laplacian of Gaussian) detector option (Tinevez et al., 2017). This was used to find the location 
of a centroid of a immobile bright spot in the worm, which could be used as reference point. Using the position of this spot in each time point of the raw movie (M1), we generated a new movie (M2) corrected by the drift. However, this method is not robust enough due to the precision of localization of the Gaussian fit. In order to minimize the residual drift, we generated kymographs of movie M2 using the Fiji plugin KymographClear and extracted the position of the base using the intensity contrast in the kymograph (Mangeol et al., 2016). This second correction of the drift was then used to calculate the intensity in the different parts of the cilia.

\section{Correction for spontaneous photobleaching}

To correct the intensity values in the different ciliary segments for spontaneous photobleaching, the intensity values before repellent addition $\left(t<t_{0}\right)$ were fitted by a decreasing exponential curve $(A \exp (-t / \tau)+C)$. The values of the intensity at each time of the movie were then multiplied by the exponential part $\exp \left(\left(t+t_{0}\right) / \tau\right)$.

\section{Averaging over multiple cilia}

For TBB-4::EGFP and XBX-1::EGFP, the intensity values were corrected for spontaneous photobleaching and normalized by the values in the different parts at the beginning of the movies. These normalized intensities were then averaged ( $n=8$ for TBB- 4 and $n=7$ for XBX-1). For OCR-2, the intensities in the different ciliary segments were normalized by the sum of the intensity of the whole cilia. These normalized intensities were then averaged $(n=8)$.

\section{Acknowledgements}

We thank J. Mijalkovic for helpful discussion and support, and Laurent Cognet and Antoine Godin (University of Bordeaux) for help and discussions regarding the analysis of single-molecule trajectories. We acknowledge financial support from the Netherlands Organisation for Scientific Research (NWO) via a Foundation for Fundamental Research on Matter (FOM) program grant ("The Signal is the Noise") and from the European Research Council under the European Union's Horizon 2020 research and innovation programme (Grant agreement no. 788363; "HITSCIL").

\section{Author Contributions}

Conceptualization and Methodology, J.v.K. and E.J.G.P.; Investigation, JvK; Formal Analysis, J.v.K. and N.D.; Resources, J.v.K. and E.J.G.P.; Writing - Original Draft, J.v.K. and E.J.G.P.; Writing - Review \& Editing, J.v.K., N.D. and E.J.G.P.; Funding Acquisition and Supervision, E.J.G.P.

\section{Declaration of Interests}

The authors declare no competing interests. 


\section{References}

Badgandi HB, Hwang S, Shimada IS, Loriot E, Mukhopadhyay S. 2017. Tubby family proteins are adapters for ciliary trafficking of integral membrane proteins $1-18$.

Behringer DC, Karvonen A, Bojko J. 2018. Parasite avoidance behaviours in aquatic environments. Philos Trans R Soc B Biol Sci 373. doi:10.1098/rstb.2017.0202

Benov L, Fridovich I. 1996. Escherichia coli exhibits negative chemotaxis in gradients of hydrogen peroxide, hypochlorite, and N-chlorotaurine: Products of the respiratory burst of phagocytic cells. Proc Natl Acad Sci U S A 93:4999-5002. doi:10.1073/pnas.93.10.4999

Blacque OE, Reardon MJ, Li C, Mccarthy J, Mahjoub MR, Ansley SJ, Badano JL, Mah AK, Beales PL, Davidson WS, Johnsen RC, Audeh M, Plasterk RHA, Baillie DL, Katsanis N, Quarmby LM, Wicks SR, Leroux MR. 2004. Loss of C . elegans BBS-7 and BBS-8 protein function results in cilia defects and compromised intraflagellar transport. Genes Dev 18:1630-1642. doi:10.1101/gad.1194004.copeptide

Brear AG, Yoon J, Wojtyniak M, Sengupta P. 2014. Diverse cell type-specific mechanisms localize G protein-coupled receptors to Caenorhabditis elegans sensory cilia. Genetics 197:667-684. doi:10.1534/genetics.114.161349

Brenner S. 1974. The genetics of Caenorhabditis elegans. Genetics 77:71-94.

Chou HT, Apelt L, Farrell DP, White SR, Woodsmith J, Svetlov V, Goldstein JS, Nager AR, Li Z, Muller J, Dollfus H, Nudler E, Stelzl U, DiMaio F, Nachury M V., Walz T. 2019. The Molecular Architecture of Native BBSome Obtained by an Integrated Structural Approach. Structure 27:13841394.e4. doi:10.1016/j.str.2019.06.006

Chronis N, Zimmer M, Bargmann CI. 2007. Microfluidics for in vivo imaging of neuronal and behavioral activity in Caenorhabditis elegans 4:727-731. doi:10.1038/NMETH1075

Collingridge P, Brownlee C, Wheeler GL. 2013. Compartmentalized calcium signaling in cilia regulates intraflagellar transport. Curr Biol 23:2311-2318. doi:10.1016/j.cub.2013.09.059

Culotti JG, Russell RL. 1978. Osmotic avoidance defective mutants of the nematode Caenorhabditis elegans. Genetics 90:243-256.

Danné N. 2018. Etude de la structure nanométrique et de la viscosité locale de l'espace extracellulaire du cerveau par microscopie de fluorescence de nanotubes de carbone uniques. Laboratoire Photonique, Numérique et Nanosciences.

Davies AG, Pierce-Shimomura JT, Kim H, VanHoven MK, Thiele TR, Bonci A, Bargmann CI, McIntire SL. 2003. A Central Role of the BK Potassium Channel in Behavioral Responses to Ethanol in C. elegans. Cell 115:655-666. doi:10.1016/S0092-8674(03)00979-6

Dwyer ND, Troemel ER, Sengupta P, Bargmann CI. 1998. Odorant receptor localization to olfactory cilia is mediated by ODR-4, a novel membrane-associated protein. Cell 93:455-466. doi:10.1016/S0092-8674(00)81173-3

Edwards SW, Tan CM, Limbird LE. 2000. Localization of G-protein-coupled receptors in health and disease. Trends Pharmacol Sci 21:304-308. doi:10.1016/S0165-6147(00)01513-3

Engel BD, Ishikawa H, Wemmer KA, Geimer S, Wakabayashi KI, Hirono M, Craige B, Pazour GJ, 
Witman GB, Kamiya R, Marshall WF. 2012. The role of retrograde intraflagellar transport in flagellar assembly, maintenance, and function. J Cell Biol 199:151-167. doi:10.1083/jcb.201206068

Frøkjær-Jensen C, Wayne Davis M, Hopkins CE, Newman BJ, Thummel JM, Olesen SP, Grunnet M, Jorgensen EM. 2008. Single-copy insertion of transgenes in Caenorhabditis elegans. Nat Genet 40:1375-1383. doi:10.1038/ng.248

Gal N, Lechtman-Goldstein D, Weihs D. 2013. Particle tracking in living cells: A review of the mean square displacement method and beyond. Rheol Acta 52:425-443. doi:10.1007/s00397-013-0694-6

Garcia-Gonzalo FR, Reiter JF. 2017. Open Sesame: How transition fibers and the transition zone control ciliary composition. Cold Spring Harb Perspect Biol 9. doi:10.1101/cshperspect.a028134

Godin AG, Varela JA, Gao Z, Danné N, Dupuis JP, Lounis B, Groc L, Cognet L. 2017. Single-nanotube tracking reveals the nanoscale organization of the extracellular space in the live brain. Nat Nanotechnol 12:238-243. doi:10.1038/nnano.2016.248

Goetz SC, Anderson K V. 2010. The primary cilium: A signalling centre during vertebrate development. Nat Rev Genet 11:331-344. doi:10.1038/nrg2774

Gruntman M, Groß D, Májeková M, Tielbörger K. 2017. Decision-making in plants under competition. Nat Commun 8:1-8. doi:10.1038/s41467-017-02147-2

Hendry TA, Ligon RA, Besler KR, Fay RL, Smee MR. 2018. Visual Detection and Avoidance of Pathogenic Bacteria by Aphids. Curr Biol 28:3158-3164.e4. doi:10.1016/j.cub.2018.07.073

Hilliard MA, Apicella AJ, Kerr R, Suzuki H, Bazzicalupo P, Schafer WR. 2005. In vivo imaging of C . elegans ASH neurons : cellular response and adaptation to chemical repellents 24:63-72. doi:10.1038/sj.emboj.7600493

Hilliard MA, Bargmann CI, Bazzicalupo P. 2002. C. elegans Responds to Chemical Repellents by Integrating Sensory Inputs from the Head and the Tail. Curr Biol 12:730-734.

Hilliard MA, Bergamasco C, Arbucci S, Plasterk RH, Bazzicalupo P. 2004. Worms taste bitter: ASH neurons, QUI-1, GPA-3 and ODR-3 mediate quinine avoidance in Caenorhabditis elegans. EMBO J23:1101-1111. doi:10.1038/sj.emboj.7600107

Holcman D, Parutto P, Chambers JE, Fantham M, Young LJ, Marciniak SJ, Kaminski CF, Ron D, Avezov E. 2018. Single particle trajectories reveal active endoplasmic reticulum luminal flow. Nat Cell Biol 20:1118-1125. doi:10.1038/s41556-018-0192-2

Huang K, Diener DR, Mitchell A, Pazour GJ, Witman GB, Rosenbaum JL. 2007. Function and dynamics of PKD2 in Chlamydomonas reinhardtii flagella. J Cell Biol 179:501-514. doi:10.1083/jcb.200704069

Iii GG, Raleigh DR, Reiter JF. 2018. How the Ciliary Membrane Is Organized Inside-Out to Communicate Outside-In. Curr Biol 28:R421-R434. doi:10.1016/j.cub.2018.03.010

Imaging R, Woldemariam S, Nagpal J, Hill T, Li J, Schneider MW, Shankar R, Tran A, Costa WS, Krzyzanowski MC, Yu Y V, Gottschalk A, Etoile NL. 2019. Using a Robust and Sensitive GFPBased cGMP Sensor for Real-Time Imaging in Intact Caenorhabditis elegans. Genetics 213:59-77.

Jansen G, Thijssen KL, Werner P, van der Horst M, Hazendonk E, Plasterk RH. 1999. The complete family of genes encoding G proteins of Caenorhabditis elegans. Nat Genet 21:414-9. 
doi:10.1038/7753

Jaqaman K, Loerke D, Mettlen M, Kuwata H, Grinstein S, Schmid SL, Danuser G. 2008. Robust singleparticle tracking in live-cell time-lapse sequences. Nat Methods 5:695-702. doi:10.1038/NMETH.1237

Jiang JY, Falcone JL, Curci S, Hofer AM. 2019. Direct visualization of cAMP signaling in primary cilia reveals up-regulation of ciliary GPCR activity following Hedgehog activation. Proc Natl Acad Sci 201819730. doi:10.1073/pnas.1819730116

Kaplan OI, Molla-Herman A, Cevik S, Ghossoub R, Kida K, Kimura Y, Jenkins P, Martens JR, Setou M, Benmerah A, Blacque OE. 2010. The AP-1 clathrin adaptor facilitates cilium formation and functions with RAB-8 in C. elegans ciliary membrane transport. J Cell Sci 123:3966-3977. doi: $10.1242 /$ jcs. 073908

Klink BU, Gatsogiannis C, Hofnagel O, Wittinghofer A. 2019. Structure of the human BBSome core complex in the open conformation. bioRxiv 1-36.

Kozminski KG, Johnson K a, Forscher P, Rosenbaum JL. 1993. A motility in the eukaryotic flagellum unrelated to flagellar beating. Proc Natl Acad Sci USA 90:5519-5523.

Lechtreck KF. 2015. IFT-cargo interactions and protein transport in cilia. Trends Biochem Sci 40:765778. doi:10.1016/j.physbeh.2017.03.040

Lechtreck KF, Brown JM, Sampaio JL, Craft JM, Shevchenko A, Evans JE, Witman GB. 2013. Cycling of the signaling protein phospholipase $\mathrm{D}$ through cilia requires the BBSome only for the export phase. $J$ Cell Biol 201:249-261. doi:10.1083/jcb.201207139

Mangeol P, Prevo B, Peterman EJG. 2016. KymographClear and KymographDirect : two tools for the automated quantitative analysis of molecular and cellular dynamics using kymographs. Mol Biol Cell 27:1948-1957. doi:10.1091/mbc.E15-06-0404

Marshall WF, Rosenbaum JL. 2001. Intraflagellar transport balances continuous turnover of outer doublet microtubules: Implications for flagellar length control. J Cell Biol 155:405-414. doi: $10.1083 /$ jcb.200106141

Mijalkovic J, Girard J, van Krugten J, van Loo J, Zhang Z, Loseva E, Oswald F, Peterman EJG. 2020. Cutting off ciliary protein import: Intraflagellar transport after dendritic femtosecond-laser ablation. Mol Biol Cell. doi:10.1091/mbc.e18-06-0399

Mijalkovic J, Prevo B, Oswald F, Mangeol P, Peterman EJG. 2017. Ensemble and single-molecule dynamics of IFT dynein in Caenorhabditis elegans cilia. Nat Commun 8:1-9. doi:10.1038/ncomms 14591

Mijalkovic J, van Krugten J, Oswald F, Acar S, Peterman EJG. 2018. Single-Molecule Turnarounds of Intraflagellar Transport at the C. elegans Ciliary Tip. Cell Rep 25:1701-1707.e2. doi:10.1016/j.celrep.2018.10.050

Milenkovic L, Weiss LE, Yoon J, Roth TL, Su YRS, Sahl SJ, Scott MP, Moerner WE. 2015. Singlemolecule imaging of Hedgehog pathway protein Smoothened in primary cilia reveals binding events regulated by Patched1. Proc Natl Acad Sci U S A 112:8320-8325. doi:10.1073/pnas.1510094112

Moore RS, Kaletsky R, Murphy CT. 2019. Piwi/PRG-1 Argonaute and TGF- $\beta$ Mediate Transgenerational Learned Pathogenic Avoidance. Cell 177:1827-1841.e12. 
doi:10.1016/j.cell.2019.05.024

Mukhopadhyay S, Wen X, Chih B, Nelson CD, Lane WS, Scales SJ, Jackson PK. 2010. TULP3 bridges the IFT-A complex and membrane phosphoinositides to promote trafficking of $\mathrm{G}$ protein-coupled receptors into primary cilia. Genes Dev 24:2180-2193. doi:10.1101/gad.1966210

Nachury M V., Mick DU. 2019. Establishing and regulating the composition of cilia for signal transduction. Nat Rev Mol Cell Biol. doi:10.1038/s41580-019-0116-4

Oswald F, Prevo B, Acar S, Peterman EJG. 2018. Interplay between Ciliary Ultrastructure and IFT-Train Dynamics Revealed by Single-Molecule Super-resolution Imaging. Cell Rep 25:224-235. doi:10.1016/j.celrep.2018.09.019

Oswald F, Varadarajan A, Lill H, Peterman EJG, Bollen YJM. 2016. MreB-Dependent Organization of the E. coli Cytoplasmic Membrane Controls Membrane Protein Diffusion. Biophys J 110:1139_ 1149. doi:10.1016/j.bpj.2016.01.010

Paix A, Folkmann A, Seydoux G. 2017. Precision genome editing using CRISPR-Cas9 and linear repair templates in C. elegans. Methods 121-122:86-93. doi:10.1016/j.ymeth.2017.03.023

Pazour GJ, Dickert BL, Witman GB. 1999. The DHC1b (DHC2) isoform of cytoplasmic dynein is required for flagellar assembly. J Cell Biol 144:473-481. doi:10.1083/jcb.144.3.473

Perkins LA, Hedgecock EM, Thomson JN, Culotti JG. 1986. Mutant sensory cilia in the nematode C. elegans. Dev Biol 117:456-487.

Pifferi S, Boccaccio A, Menini A. 2006. Cyclic nucleotide-gated ion channels in sensory transduction. FEBS Lett 580:2853-2859. doi:10.1016/j.febslet.2006.03.086

Pigino G, Geimer S, Lanzavecchia S, Paccagnini E, Cantele F, Diener DR, Rosenbaum JL, Lupetti P. 2009. Electron-tomographic analysis of intraflagellar transport particle trains in situ. J Cell Biol 187:135-148. doi:10.1083/jcb.200905103

Prevo B, Mangeol P, Oswald F, Scholey JM, Peterman EJG. 2015. Functional differentiation of cooperating kinesin-2 motors orchestrates cargo import and transport in C. elegans cilia. Nat Cell Biol 17:1536-1545. doi:10.1038/ncb3263

Qin H, Burnette DT, Bae Y, Forscher P, Barr MM, Rosenbaum JL, Haven N. 2005. Intraflagellar Transport Is Required for the Vectorial Movement of TRPV Channels in the Ciliary Membrane. Curr Biol 15:1695-1699. doi:10.1016/j.cub.2005.08.047

Rahman A, Brown CW. 1983. Effect of $\mathrm{pH}$ on the critical micelle concentration of sodium dodecyl sulphate. J Appl Polym Sci 28:1331-1334.

Renner M, Domanov Y, Sandrin F, Izeddin I, Bassereau P, Triller A. 2011. Lateral diffusion on tubular membranes: Quantification of measurements bias. PLoS One 6. doi:10.1371/journal.pone.0025731

Saxton MJ. 1994. Anomalous diffusion due to obstacles: a Monte Carlo study. Biophys J 66:394-401. doi:10.1016/S0006-3495(94)80789-1

Scholey JM. 2003. Intraflagellar Transport. Annu Rev Cell Dev Biol 19:423-443. doi:10.1146/annurev.cellbio.19.111401.091318

Schou KB, Pedersen LB, Christensen ST. 2015. Ins and outs of GPCR signaling in primary cilia. EMBO 

transport drives flagellar surface motility. Elife 2013:1-19. doi:10.7554/eLife.00744

Signor D, Wedaman KP, Rose LS, Scholey JM. 1999. Two heteromeric kinesin complexes in chemosensory neurons and sensory cilia of Caenorhabditis elegans. Mol Biol Cell 10:345-360. doi:10.1091/mbc.10.2.345

800

801

802

803

804

805

806

807

808

809

810

811

812

813

814

815

Snow JJ, Ou G, Gunnarson AL, Walker MRS, Zhou HM, Brust-mascher I, Scholey JM. 2004. Two anterograde intraflagellar transport motors cooperate to build sensory cilia on $\mathrm{C}$. elegans neurons. Nat Cell Biol 6:1109-1113. doi:10.1038/ncb1186

Tinevez JY, Perry N, Schindelin J, Hoopes GM, Reynolds GD, Laplantine E, Bednarek SY, Shorte SL, Eliceiri KW. 2017. TrackMate: An open and extensible platform for single-particle tracking. Methods 115:80-90. doi:10.1016/j.ymeth.2016.09.016

Tobin DM, Madsen DM, Kahn-kirby A, Peckol EL, Moulder G, Barstead R, Maricq A V, Bargmann CI, Francisco S. 2002. Combinatorial Expression of TRPV Channel Proteins Defines Their Sensory Functions and Subcellular Localization in C . elegans Neurons 35:307-318.

Tobin JL, Beales PL. 2009. The nonmotile ciliopathies. Genet Med 11:386-402. doi:10.1097/GIM.0b013e3181a02882

Tran A, Tang A, Loughlin CTO, Balistreri A, Chang E, Villa DC, Li J, Varshney A, Jimenez V, Pyle J, Tsujimoto B, Wellbrook C, Vargas C, Duong A, Ali N, Matthews SY, Levinson S, Woldemariam S, Khuri S, Bremer M, Eggers DK, Etoile NL, Conrad LCM, Vanhoven MK. 2017. C . elegans avoids toxin-producing Streptomyces using a seven transmembrane domain chemosensory receptor. Elife 6:1-16.

Van Den Wildenberg SMJL, Bollen YJM, Peterman EJG. 2011. How to quantify protein diffusion in the bacterial membrane. Biopolymers 95:312-321. doi:10.1002/bip.21585

van Krugten J, Peterman EJG. 2018. Single-Molecule Fluorescence Microscopy in Living Caenorhabditis elegans, Single Molecule Analysis. doi:10.1007/978-1-4939-7271-5

Ward S. 1973. Chemotaxis by the nematode Caenorhabditis elegans: identification of attractants and analysis of the response by use of mutants. Proc Natl Acad Sci U S A 70:817-821. doi:10.1073/pnas.70.3.817

Weiss LE, Milenkovic L, Yoon J, Stearns T, Moerner WE. 2019. Motional dynamics of single Patched1 molecules in cilia are controlled by Hedgehog and cholesterol. Proc Natl Acad Sci U S A 116:55505557. doi:10.1073/pnas. 1816747116

Ye F, Breslow DK, Koslover EF, Spakowitz AJ, James Nelson W, Nachury M V. 2013. Single molecule imaging reveals a major role for diffusion in the exploration of ciliary space by signaling receptors. Elife 2013:1-16. doi:10.7554/eLife.00654.001

Ye F, Nager AR, Nachury M V. 2018. BBSome trains remove activated GPCRs from cilia by enabling passage through the transition zone. J Cell Biol 217:1847-1868. 


\section{Supplemental information}

\section{Nematodes experience prolonged exposure to SDS during repellent experiments}

To investigate whether the time course of SDS concentration around the imaged worms might be the cause of the delay, we added fluorescein in an identical way as SDS and measured its intensity over time. Following an immediate increase of fluorescein intensity, which explains the immediate avoidance response, a plateau was reached after about 2 minutes. This indicates that a low concentration is sufficient to evoke an avoidance response.

842

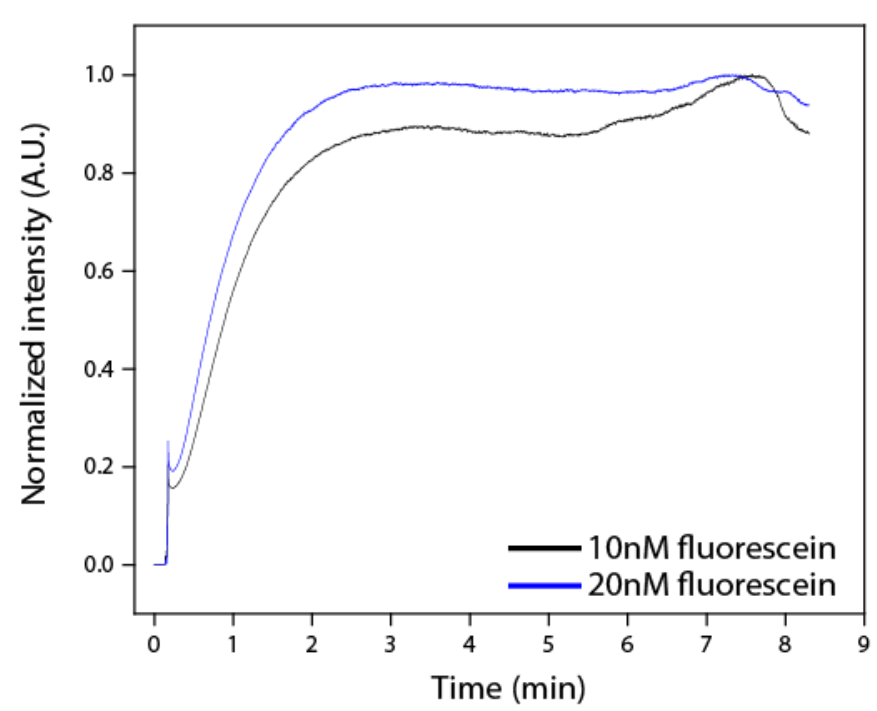

843 Figure S1: Intensity of fluorescein over time, after addition to sample. A $5 \mu$ droplet of fluorescein 844 (of $10 \mathrm{nM}$ and $20 \mathrm{nM}$ ) was added to an agarose pad on a cover slip, and the intensity measured over time. 


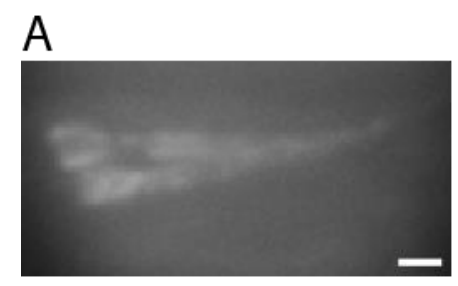

B
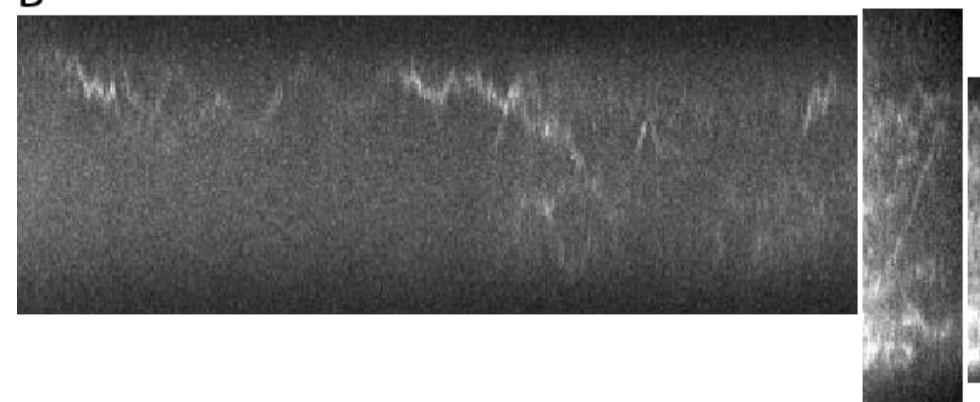

tip

845 Figure S2: Low expression levels of GPCR SRB-6 in phasmid cilia. (A) Time-averaged image

846 sequence shows the ciliary distribution of SRB-6::EGFP is comparable to that of OCR-2::EGFP (Scale bar

$8471 \mu \mathrm{m}$ ). (B) Kymographs showing salutatory movement and active transport of SRB-6 in the phasmid cilia

848 (horizontal: time, 2 s; vertical: distance, scale bar $2 \mu \mathrm{m}$ ). Related to Movie S2. 
bioRxiv preprint doi: https://doi.org/10.1101/2020 11.19.390005; this version posted November 20, 2020. The copyright holder for this preprint (which was not certified by peer review) is the author/funder, who has granted bioRxiv a license to display the preprint in perpetuity. It is made available under aCC-BY-NC 4.0 International license.
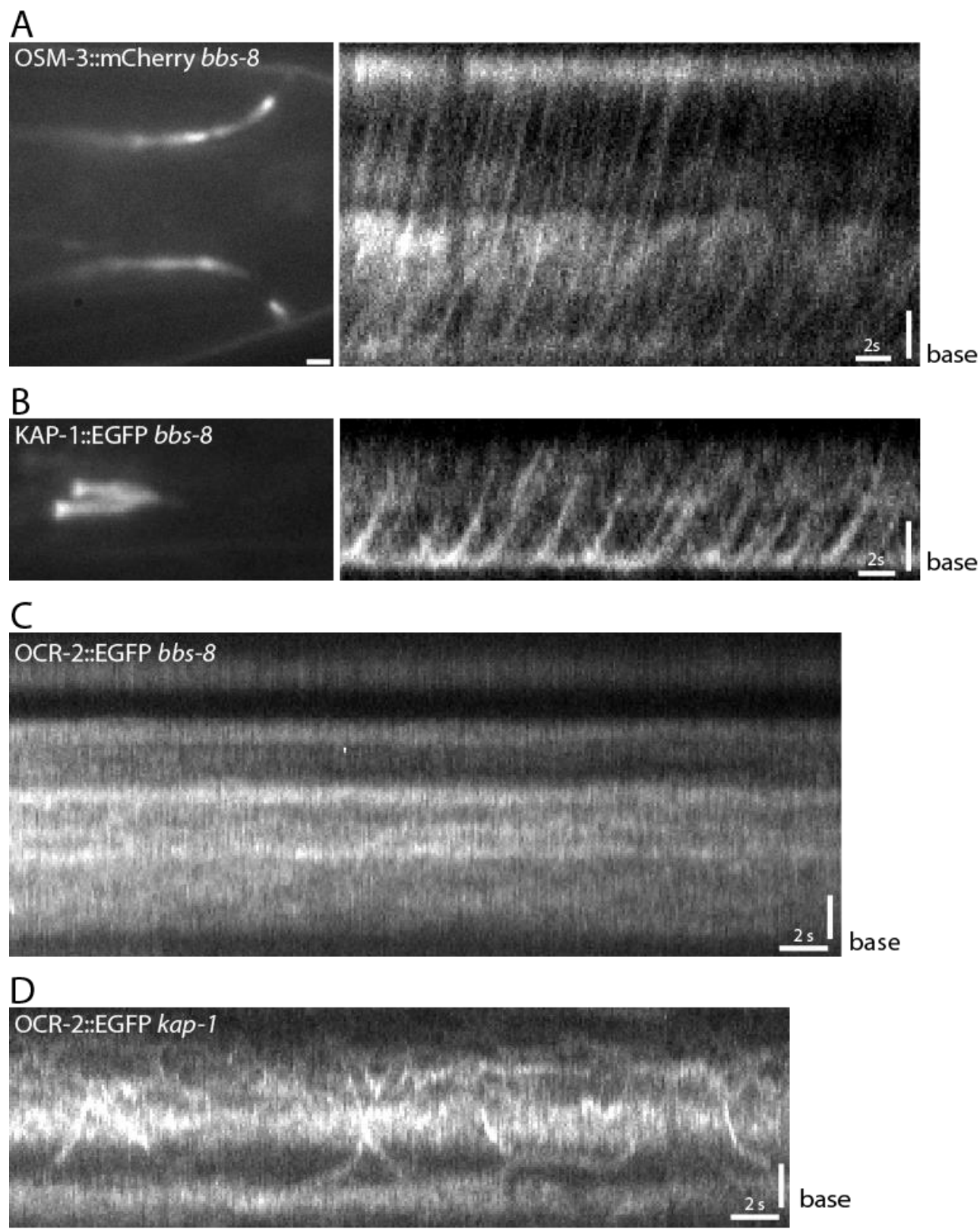

849 Figure S3: Functional IFT in cilia lacking functional BBSome. Time-average image sequences and kymographs of OSM-3::EGFP (A) and KAP-1::EGFP (B) in $b b s-8$ background show functional IFT, 851 despite being chemotaxis deficient. These data indicate that although the distribution of OCR-2 in $b b s-8$ 852 worms is drastically different, the cilia itself are still intact, and IFT is functional, though not coupled to 853 OCR-2. (C) Kymograph of OCR-2::EGFP in bbs-8 background, showing no IFT movements of OCR-2. 854 (D) Kymograph of OCR-2::EGFP in kap-1 mutant background (horizontal: time, 2 s; vertical: scale bar 1 $855 \mu \mathrm{m})$. 
bioRxiv preprint doi: https://doi.org/10.1101/2020.11.19.390005; this version posted November 20, 2020. The copyright holder for this preprint (which was not certified by peer review) is the author/funder, who has granted bioRxiv a license to display the preprint in perpetuity. It is made available under aCC-BY-NC 4.0 International license.

\section{OCR-2 is transported towards the cilia via the trans-golgi network.}
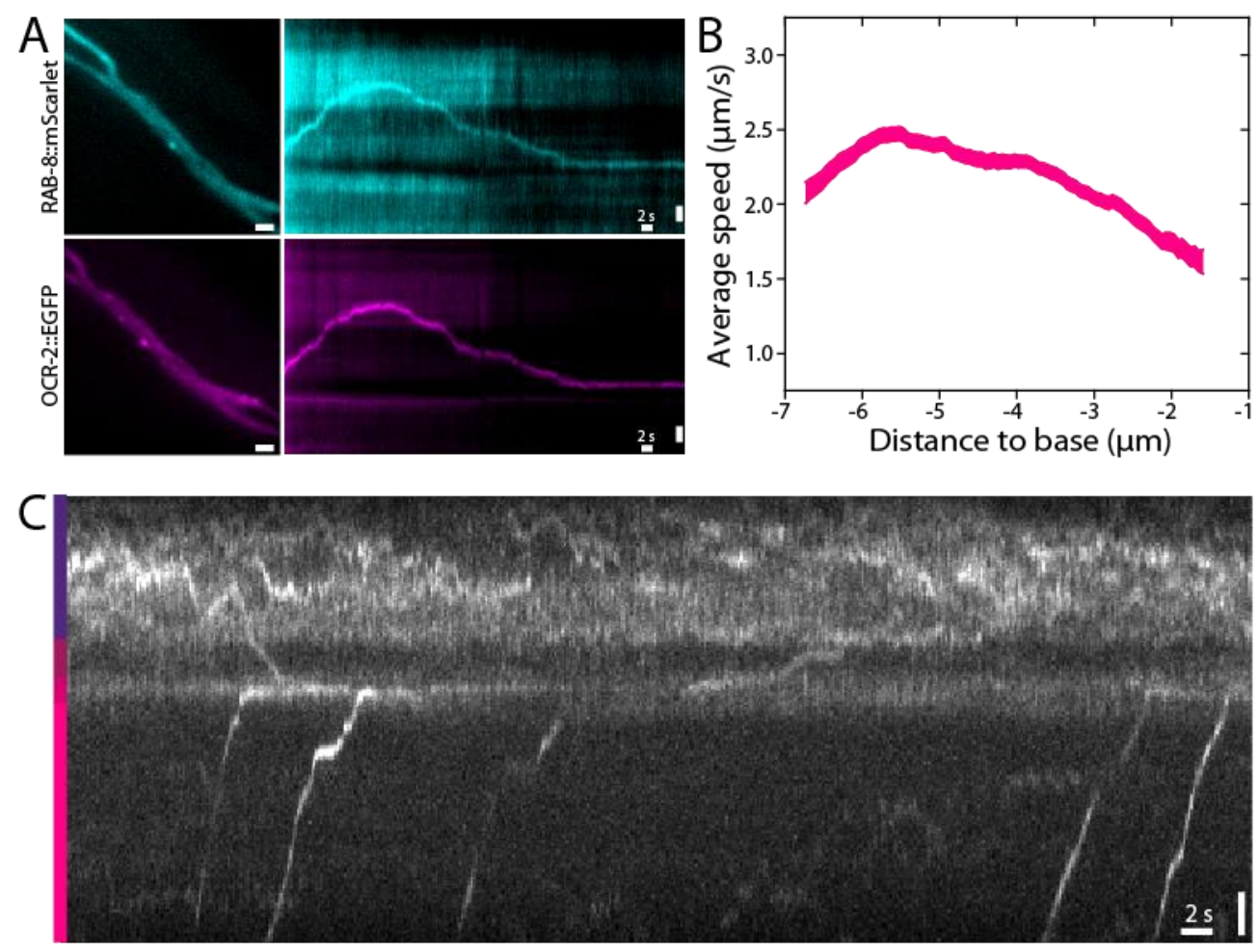

Figure S4: Dendritic transport of OCR-2 towards phasmid cilia. (A) Co-localization, as shown in a frame of the movie (left) and on a kymograph of the dendrite (right), of vesicle marker RAB-8 (cyan, top) and OCR-2 (magenta, bottom) in foci travelling in the phasmid neuron dendrite. (B) Average speed of OCR-2::EGFP foci traveling towards the cilium. (C) Kymograph showing OCR-2::EGFP foci arriving in the PCMC. Here, the signal gradually decreases, indicating docking and diffusion of OCR-2 with and in the membrane of the PCMC. A retrograde and anterograde TZ crossing can also be observed. Color bar on the left indicates the dendrite (bottom), PCMC, TZ and proximal segment (top) according to the 

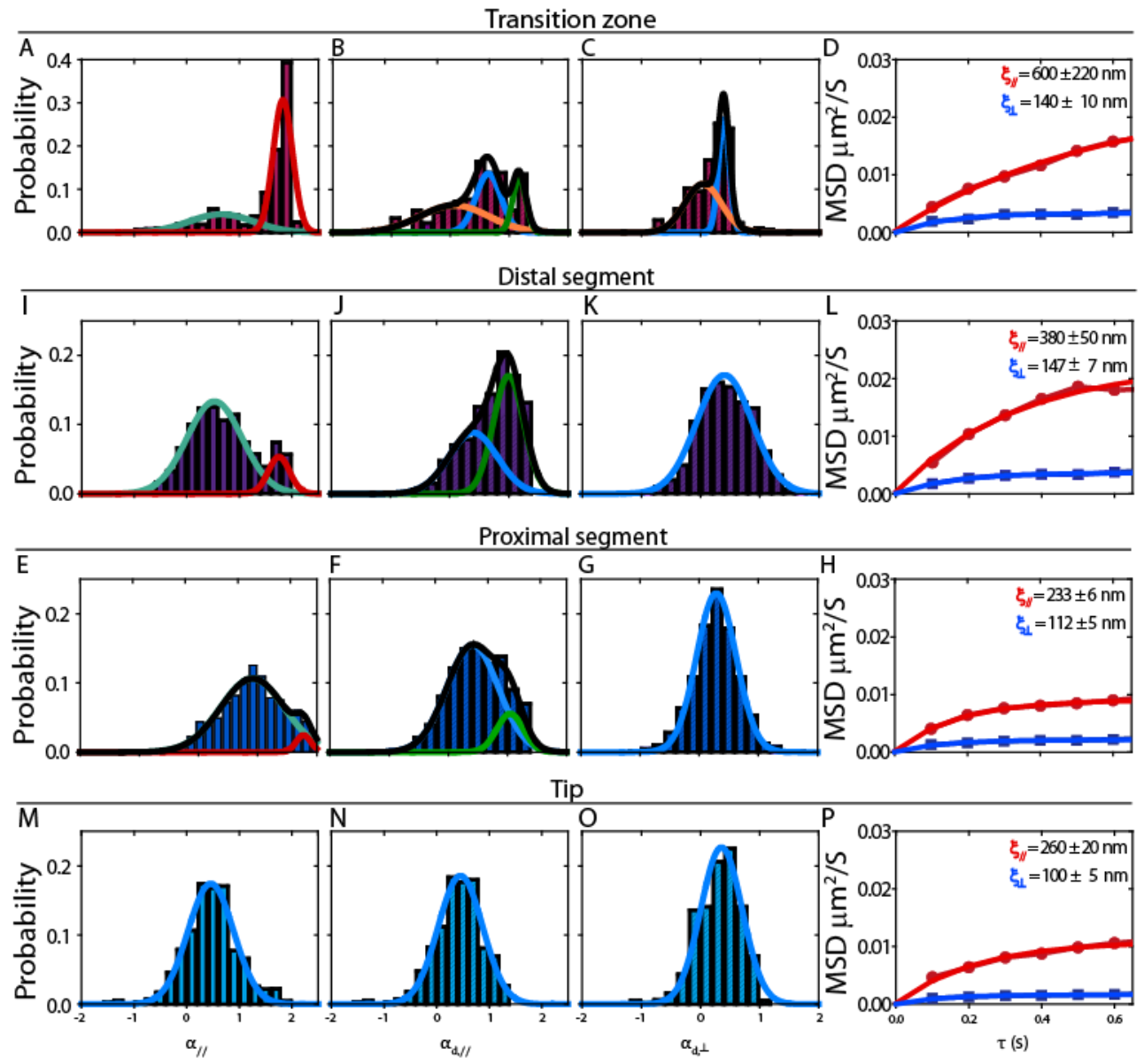

Q

\begin{tabular}{|c|c|c|c|c|c|c|c|c|c|c|c|c|c|c|c|}
\hline & \multicolumn{3}{|c|}{ TZ } & \multicolumn{3}{|c|}{ PS } & \multicolumn{3}{c|}{ DS } & \multicolumn{3}{|c|}{ Tip } & \multicolumn{3}{c|}{ All } \\
\hline & mean & std & $\%$ & mean & std & $\%$ & mean & std & $\%$ & mean & std & $\%$ & mean & std & $\%$ \\
\hline$\alpha_{/ /, t}$ & 1.82 & 0.18 & 70 & 1.78 & 0.11 & 3 & 1.74 & 0.20 & 14 & & & & 1.78 & 0.17 & 16 \\
\hline$\alpha_{/ /, s+d}$ & 0.65 & 0.59 & 30 & 0.94 & 0.54 & 97 & 0.53 & 0.53 & 86 & 0.46 & 0.43 & 100 & 0.66 & 0.56 & 84 \\
\hline$\alpha_{/ /, d}$ & 1.17 & 0.12 & 18 & 1.02 & 0.24 & 49 & 1.04 & 0.23 & 17 & & & & & & \\
\hline$\alpha_{/ /, s}$ & 0.65 & 0.21 & 37 & 0.47 & 0.43 & 51 & 0.41 & 0.43 & 83 & 0.46 & 0.43 & 100 & & & \\
\hline$\alpha_{\perp}$ & 0.39 & 0.09 & 40 & 0.41 & 0.47 & 100 & 0.29 & 0.33 & 100 & 0.36 & 0.35 & 100 & 0.38 & 0.40 & 100 \\
\hline
\end{tabular}

Figure S5: Summary of $\boldsymbol{\alpha}$ values and extraction of the sizes explored during subdiffusion. (A, E, I and M) Histograms of $\alpha / /$ values in TZ, PS, DS and Tip respectively from all the trajectories. Straight curves indicate Gaussian fits of histogram showing transported and subdiffusion fractions, (B, F, J, N) Histograms of $\alpha_{/ /}$values when $\alpha_{/ /}<1.4$ in TZ, PS, DS and Tip respectively. Straight curves indicate Gaussian fits of histogram showing diffusion and subdiffusion fractions, (C, G, K, O) Histograms of $\alpha_{\perp}$ values when $\alpha_{/ /}<1.4$ in TZ, PS, DS and Tip respectively. Straight curves indicate Gaussian fits of histogram showing diffusion and subdiffusion fractions, (D, H, L, P) Mean Square Displacement in function of the lag time in the parallel and perpendicular directions plotted for lower than unity values of $\alpha_{/ /}$(blue circles) and $\alpha_{\perp}$ (red squares) respectively (with a range \pm 0.05 ). Blue and Red curve indicated fits of MSD with the equation $M S D_{/ /, \perp}=\frac{\xi_{/ /, \perp}{ }^{2}}{6}\left(1-\frac{96}{\pi^{4}} \exp \left(-\frac{\pi^{2}}{4} \frac{\mathrm{D}_{/ /, \perp} \tau}{\left(\xi_{/ /, \perp} / 2\right)^{2}}\right)\right)$. Values of sizes explored by OCR-2 $\left(\xi_{/ /, \perp}\right)$ are extracted from fits. Table showing all $\alpha_{/ /, \perp}$ values extracted from Gaussian fits of histograms (A-C, E-G, I-K, M-O). 


\section{Supplemental tables}

\begin{tabular}{|c|c|c|}
\hline Strain & Genotype & Short notation \\
\hline EJP212 & $\begin{array}{l}\text { vuaSi26 [pJM6; Pxbx-1::xbx-1::EGFP; cb-unc- } \\
119(+) \text { ] I; vuaSi2 [pBP22; Posm-3::osm- } \\
\text { 3::mCherry; cb-unc-119(+)] II; osm-3(p802) IV; } \\
\text { xbx-1(ok279) V }\end{array}$ & XBX-1::EGFP and OSM-3::mCherry \\
\hline EJP401 & $\begin{array}{l}\text { vuaSi401 [pSA401; Ptbb-4::tbb-4::eGFP;cb-unc- } \\
119(+)] I\end{array}$ & TBB-4::EGFP \\
\hline EJP70 & $\begin{array}{l}\text { mks-6(gk674) I; vuaSi21 [pBP39; Pmks-6::mks- } \\
\text { 6::mCherry; cb-unc-119(+)] II; kap-1(ok676) III; } \\
\text { vuaSi1 [pBP20; Pkap-1::kap-1::eGFP; cb-unc- } \\
119(+)] \text { IV }\end{array}$ & KAP-1::EGFP and MKS-6::mCherry \\
\hline EJP48 & $\begin{array}{l}\text { vuaSi1 [pBP20; Pkap-1::kap-1::eGFP; cb-unc- } \\
\text { 119(+)] I; vuaSi2 [pBP22; Posm-3::osm- } \\
\text { 3::mCherry; cb-unc-119(+)] II; kap-1(ok676) III; } \\
\text { osm-3(p802) IV; bbs-8(nx77) V }\end{array}$ & $\begin{array}{l}\text { KAP-1::EGFP and OSM-3::mCherry, } \\
\Delta \text { bbs-8 }\end{array}$ \\
\hline EJP501 & vuaSi001 ocr-2::eGFP IV & OCR-2::EGFP \\
\hline EJP502 & kap-1(ok676) III; vuaSi001 ocr-2::eGFP IV & OCR-2::EGFP, $\Delta$ kap-1 \\
\hline EJP503 & vuaSi002 srb-6::eGFP II & SRB-6::EGFP \\
\hline EJP504 & vuaSi001 ocr-2::eGFP IV; bbs-8(nx77) V & OCR-2::EGFP, $\Delta$ bbs- 8 \\
\hline EJP505 & $\begin{array}{l}\text { vuaSi001 ocr-2::eGFP } I V ; \text { vuaEx005 [Ptbb- } \\
4:: R A B-8:: E G F P]\end{array}$ & $\begin{array}{l}\text { OCR-2::EGFP, RAB-8::mScarlet } \\
\text { (extrachromosomal array) }\end{array}$ \\
\hline EJP506 & $\begin{array}{l}\text { vuaSi001 ocr-2::eGFP IV; vuaEx006 [Ptbb- } \\
4:: M K S-8:: E G F P]\end{array}$ & $\begin{array}{l}\text { OCR-2::EGFP, MKS-6:: mScarlet } \\
\text { (extrachromosomal array) }\end{array}$ \\
\hline
\end{tabular}

Supplemental table 1: Strains used in this study. 


\begin{tabular}{|c|c|c|}
\hline Name & Sequence $\left(5^{\prime}-3^{\prime}\right)$ & Used for \\
\hline $\begin{array}{l}\text { crRNA_ocr } \\
-2 \# 2\end{array}$ & AATGGTGTTCAGAATGGAAAGUUUUAGAGCUAUGCUGUUUUG & \multirow{7}{*}{$\begin{array}{l}\text { EJP501, } \\
\text { OCR-2: : EGFP }\end{array}$} \\
\hline JvK०1F & $\begin{array}{l}\text { TCGAGGGGCTGGTGGAGGTGCCAATGGTGTCCAAAACGGTAACGGAGCCGCCCATG } \\
\text { GAGCATCGGGAGCCTCAGG }\end{array}$ & \\
\hline JVK๑1R & GATGGAAGGAAACAAAAAAGAGAAAAATGGTTAAATTCACTTGTAGAGCTCGTCC & \\
\hline $\mathrm{JvK} \odot 2 \mathrm{~F}$ & GCCATGATGACACGTACATATG & \\
\hline JvK@2R & CTTCAAGCAGCTCTCATTCC & \\
\hline JvK॰3F & GTCAACGGACACAAGTTCTC & \\
\hline JvKO3R & GTGACGAACTCCTTGAGGAC & \\
\hline JM42F & TCCTACAATTAGTGCAGAATTAGGG & \multirow{2}{*}{$\begin{array}{c}\text { EJP5०2, } \\
\text { OCR-2: : EGFP, } \\
\Delta \text { kap-1 }\end{array}$} \\
\hline JM42R & CCTCCATTCACCACCATTTTTGG & \\
\hline $\begin{array}{l}\text { crRNA_srb } \\
-6 \# 1\end{array}$ & GGAAGACTAAGTCATCAGGAGUUUUAGAGCUAUGCUGUUUUG & \multirow{5}{*}{$\begin{array}{l}\text { EJP503, } \\
\text { SRB-6: : EGFP }\end{array}$} \\
\hline JvK๑4F & $\begin{array}{l}\text { GAGGCTATTTTAAATGTTTGGAAGACTAAGAGCAGCGGCAGAAAGGGAGCATCGGG } \\
\text { AGCCTCAGG }\end{array}$ & \\
\hline JvK०4R & $\begin{array}{l}\text { TTGTCGTGTAATATATTTTTTCAATTATAAATCAGTCACTTGTAGAGCTCGTCCAT } \\
\text { TCCGTG }\end{array}$ & \\
\hline JvK05F & CATGGCACTTGTCTATTTCTTCTTC & \\
\hline JvK@5R & TGATCTTACGGAACTATTGACTCTC & \\
\hline JvK०6F & TAGCAAACGACAATTTCCAATAGCC & \multirow{2}{*}{$\begin{array}{c}\text { EJP5०4, } \\
\text { OCR-2: : EGFP, } \\
\Delta \text { bbs }-8\end{array}$} \\
\hline JvK๑6R & ACCCATTGGATCAGGTATCGATTT & \\
\hline JvKO7F & $\begin{array}{l}\text { CGTATCTGTGAAATAATATGATTAAATTTCAGAAGATGGCAAAAACTTACGACTAC } \\
\text { TTG }\end{array}$ & \multirow{2}{*}{$\begin{array}{c}\text { EJP505, } \\
\text { Gibson } \\
\text { assembly for } \\
\text { MKS-6: : EGFP } \\
\text { (extrachromos } \\
\text { omal array) }\end{array}$} \\
\hline JvK@7R & ATCGATGCTCCTGAGGCTCCCGATGCTCCGCCGGCAAGCAAATTGCAGCTCCAG & \\
\hline JvK०8F & $\begin{array}{l}\text { CTGTGAAATAATATGATTAAATTTCAGAAGCCGGCGGAGCATCGGGAGCCTCAGGA } \\
\text { GCATCGATGGTCAGCAAGGGAGAGGC }\end{array}$ & \multirow{6}{*}{$\begin{array}{c}\text { EJP506, } \\
\text { Gibson } \\
\text { assembly for } \\
\text { MKS-6: : EGFP } \\
\text { (extrachromos } \\
\text { omal array) }\end{array}$} \\
\hline JvK०8R & $\begin{array}{l}\text { ATTAGAAAAAAAACGTAGAAAATGAATTAAGCTTACTTGTAGAGCTCGTCCATTCC } \\
\text { TCC }\end{array}$ & \\
\hline JvK०9F & $\begin{array}{l}\text { TCCGCTTCCACCTCCTCCGCTTCCTCCGCCGGCTTCTGAAATTTAATCATATTATT } \\
\text { TCA }\end{array}$ & \\
\hline JvK@9R & $\begin{array}{l}\text { AGTTTGATGAGCACGATCAAGATGTGGAAGCCGGCGGAGCATCGGGAGCCTCAGGA } \\
\text { GCATCGATGGTCAGCAAGGGAGAGGC }\end{array}$ & \\
\hline JvK10F & CTGTGAAATAATATGATTAAATTTCAGAAGATGCGCAGAAATTCAGTTTCCGTTG & \\
\hline JVK10R & $\begin{array}{l}\text { TGCTCCTGAGGCTCCCGATGCTCCGCCGGCCCTTTTTGGTTTAAGAACAGCCAGAG } \\
\text { C }\end{array}$ & \\
\hline JvK11F & GTAATACGACTCACTAGTGGGCAGATCTACTATTTCTGTAACTACG & \multirow{4}{*}{$\begin{array}{c}\text { EJP507, } \\
\text { Gibson } \\
\text { assembly for } \\
\text { myristoylated } \\
\text { mMaple } \\
\text { (extrachromos } \\
\text { omal array) }\end{array}$} \\
\hline JvK11R & $\begin{array}{l}\text { CTTCCTTTTCCAATACATGATCCCATTTCTGAAATTTAATCATATTATTTCACAGA } \\
\text { TACGACACGC }\end{array}$ & \\
\hline JvK12F & $\begin{array}{l}\text { AATATGATTAAATTTCAGAAATGGGATCATGTATTGGAAAAGGAAGCAGTGTCTCC } \\
\text { AAGGTAACACTTAG }\end{array}$ & \\
\hline JvK12R & CGTAGAAAATGAATTAAGCTTAATTAAACTTGTAGAGCTCGTCCATGGAGTCG & \\
\hline
\end{tabular}

891 Supplemental table 2: Primers used in this study. 


\section{Supplementary movies}

894 Movie S1: Redistribution of IFT-dynein upon repellent exposure. Fluorescence microscopy image 895 sequence showing the reversible redistribution of IFT-dynein (XBX-1::EGFP) in the distal segments of the

896 phasmid chemosensory cilia of $C$. elegans after the addition of a droplet of $5 \mu l 0.1 \%$ (W/V) SDS. Related 897 to Figure 1.

898 Movie S2: Single-molecule imaging of SRB-6. Single-particle fluorescence image sequences of

899 endogenously labeled SRB-6, showing its low expression level in the phasmid cilia and mostly diffusive 900 and salutatory motility.

901 Movie S3: Single-molecule imaging of OCR-2. Collage of example single-molecule image sequences of 902 OCR-2::EGFP, demonstrating the diversity in OCR-2 motility. Related to Figure 3A. 